\title{
QUANTITATIVE INFORMATION FLOW UNDER GENERIC LEAKAGE FUNCTIONS AND ADAPTIVE ADVERSARIES*
}

\author{
MICHELE BOREALE AND FRANCESCA PAMPALONI
}

Università di Firenze, Dipartimento di Statistica, Informatica, Applicazioni (DiSIA), Viale Morgagni 65, I-50134 Florence, Italy.

e-mail address: michele.boreale@unifi.it

Independent researcher, Italy.

e-mail address: francesca.pampaloni@ge.com

\begin{abstract}
We put forward a model of action-based randomization mechanisms to analyse quantitative information flow (QIF) under generic leakage functions, and under possibly adaptive adversaries. This model subsumes many of the QIF models proposed so far. Our main contributions include the following: (1) we identify mild general conditions on the leakage function under which it is possible to derive general and significant results on adaptive QIF; (2) we contrast the efficiency of adaptive and non-adaptive strategies, showing that the latter are as efficient as the former in terms of length up to an expansion factor bounded by the number of available actions; (3) we show that the maximum information leakage over strategies, given a finite time horizon, can be expressed in terms of a Bellman equation. This can be used to compute an optimal finite strategy recursively, by resorting to standard methods like backward induction.
\end{abstract}

\section{INTRODUCTION}

Quantitative Information Flow (QIF) is a well-established approach to confidentiality analysis: the basic idea is measuring how much information flows from sensitive to observable data, relying on tools from Information Theory [14, 3, 12, 32, 11, 4, 27, 5, 6].

Two important issues that arise in QIF are: what measure one should adopt to quantify the leakage of confidential data, and the relationship between adaptive and non adaptive adversaries. Concerning the first issue, a long standing debate in the QIF community concerns the relative merits of leakage functions based on Shannon entropy (see e.g. [14, 4]) and min-entropy (see e.g. [32, 11]); other types of entropies are sometimes considered (see e.g. [26].) As a matter of fact, analytical results for each of these types of leakage functions have been so far worked out in a non-uniform, ad hoc fashion.

2012 ACM CCS: [Security and privacy]: Formal methods and theory of security - Formal security models.

Key words and phrases: Quantitative information flow, adaptive attackers, information theory, Bayesian decision theory.

* Extended and revised version of [8]. Corresponding author: Michele Boreale. Work partially supported by the EU project ASCENS under the FET open initiative in FP7 and by the italian PRIN project CINA.

넨임 LOGICAL METHODS IN COMPUTER SCIENCE
DOI:10.2168/LMCS-11(4:5)2015 (c) M. Boreale and F. Pampaloni (C) Creative Commons 
Concerning the second issue, one sees that, with the notable exception of [26] which we discuss later on, the treatment of confidentiality in QIF has so far been almost exclusively confined to attackers that can only passively eavesdrop on the mechanism; or, at best, obtain answers in response to queries (or actions) submitted in a non-adaptive fashion [7. By passive, we mean here attackers that can eavesdrop on messages, but not interfere with the generation process of these messages 1 . Clearly, there are situations where this model is not adequate. To mention but two: chosen plaintext/ciphertext attacks against cryptographic hardware or software; adaptive querying of databases whose records contain both sensitive and non-sensitive fields.

In this paper, we tackle both issues outlined above. We: (a) put forward a general QIF model where the leakage function is built around a generic uncertainty measure; and, (b) derive several general results on the relationship between adaptive and non adaptive adversaries in this model. In more detail, we assume that, based on a secret piece of information $X \in \mathcal{X}$, the mechanism responds to a sequence of queries/actions $a_{1}, a_{2}, \ldots\left(a_{i} \in A c t\right)$, adaptively submitted by an adversary, thus producing a sequence of answers/observations $Y \in \mathcal{Y}^{*}$. Responses to individual queries are in general probabilistic, either because of the presence of noise or by system's design. Moreover, the mechanism is stateless, thus answers are independent from one another. The adversary is assumed to know the distribution according to which $X$ has been generated (the prior) and the input-output behaviour of the mechanism. An adaptive adversary can choose the next query based on past observations, according to a predefined strategy. Once a strategy and a prior have been fixed, they together induce a probability space over sequences of observations. Observing a specific sequence provides the adversary with information that modifies his belief about $X$, possibly reducing his uncertainty. We measure information leakage as the average reduction in uncertainty. We work with a generic measure of uncertainty, $U(\cdot)$. Formally, $U(\cdot)$ is just a real-valued function over the set of probability distributions on $\mathcal{X}$, which represent possible beliefs of the adversary. Just two properties are assumed of $U(\cdot)$ : concavity and continuity. Note that leakage functions commonly employed in QIF, such as Shannon entropy, guessing entropy and error probability - the additive version of Smith's min-entropy-based vulnerability [32] - do fall in this category.

The other central theme of our study is the comparison between adaptive and the simpler non-adaptive strategies. All in all, our results indicate that, for even moderately powerful adversaries, there is no dramatic difference between the two, in terms of difficulty of analysis. A more precise account of our contributions follows.

- We put forward a general model of adaptive QIF; we identify mild general conditions on the uncertainty function under which it is possible to derive general and substantial results on adaptive QIF.

- We compare the difficulty of analyzing mechanisms under adaptive and non-adaptive adversaries. We first note that, for the class of mechanisms admitting a "succinct" syntactic description - e.g. devices specified by boolean formulae - the analysis problem is intractable (NP-hard), even if limited to very simple instances of the non-adaptive case. This essentially depends on the fact that such mechanisms can feature exponentially many

\footnotetext{
${ }^{1}$ Passivity in this sense does not rule out attackers that can try secrets, based on some form of oracle. Active attackers are also considered in approaches to quantitative integrity [15, a theme that will not be considered here.
} 
actions in the syntactic size of the description. In the general case, we show that nonadaptive finite strategies are as efficient as adaptive ones, up to an expansion factor in their length bounded by the number of distinct actions available. Practically, this indicates that, for mechanisms described in explicit form (e.g. by tables, like a DB) hence featuring an "affordable" number of actions available to the adversary, it may be sufficient to assess resistance of the mechanism against non-adaptive strategies. This is important, because simple analytical results are available for such strategies [7].

- We show that the maximum leakage over all strategies is the same for both adaptive and non-adaptive adversaries, and only depends on an indistinguishability equivalence relation over the set of secrets.

- We show that maximum leakage over all strategies over a finite horizon can be expressed in terms of a Bellman equation. This equation can be used to compute optimal finite strategies recursively. As an example, we show how to do that using Markov Decision Processes (MDP's) and backward induction.

- We finally give a Bayesian decision-theoretic justification of our definition of uncertainty function. We argue that each such function arises as a measure of expected loss; and, vice-versa, that (under a mild condition) each measure of expected loss is in fact an uncertainty function in our sense.

Structure of the Paper. Section 2 introduces the model. This is illustrated with a few examples in Section 3. The subsequent four sections 4, 5, 6, 7 discuss the results outlined in (2), (3), (4) and (5) above, respectively. Section 8 contains a few concluding remarks, discussion of related work and some directions for further research. Some technical material has been confined to three separate appendices.

\section{A MODEL OF ADAPTIVE QIF}

\subsection{Randomization mechanisms, uncertainty, strategies.}

Definition 2.1. An action-based randomization mechanism 2 is a 4 -tuple

$$
\mathcal{S}=\left(\mathcal{X}, \mathcal{Y}, \text { Act },\left\{M_{a}: a \in A c t\right\}\right),
$$

where (all sets finite and nonempty): $\mathcal{X}, \mathcal{Y}$ and Act are respectively the sets of secrets, observations and actions (or queries) and for each $a \in$ Act, $M_{a}$ is a stochastic matrix of dimensions $|\mathcal{X}| \times|\mathcal{Y}|$.

For each action $a \in A c t, x \in \mathcal{X}$ and $y \in \mathcal{Y}$, the element of row $x$ and column $y$ of $M_{a}$ is denoted by $p_{a}(y \mid x)$. Note that for each $x$ and $a$, row $x$ of $M_{a}$ defines a probability distribution over $\mathcal{Y}$, denoted by $p_{a}(\cdot \mid x)$. A mechanism $\mathcal{S}$ is deterministic if each entry of each $M_{a}$ is either 0 or 1 . Note that to any deterministic mechanism there corresponds a function $f: \mathcal{X} \times A c t \rightarrow \mathcal{Y}$ defined by $f(x, a)=y$, where $p_{a}(y \mid x)=1$. Recall that a real function $F$ defined over a convex subset $C \subseteq \mathbb{R}^{n}$ is concave if, for each $\lambda \in[0,1]$ and $u, v \in C$, it holds true that $F(\lambda u+(1-\lambda) v) \geq \lambda F(u)+(1-\lambda) F(v)$. In the rest of the

\footnotetext{
${ }^{2}$ The term information hiding system is sometimes found in the literature to indicate randomization mechanisms. This term, however, is also used with a rather different technical meaning in the literature on watermarking; so we prefer to avoid it altogether here.
} 
paper, we let $\mathcal{P}$ denote the set of all probability distributions on $\mathcal{X}$; this can of course be seen as a convex subset of $\mathbb{R}^{|\mathcal{X}|}$ (the probability simplex.)

Definition 2.2 (Uncertainty). A function $U: \mathcal{P} \rightarrow \mathbb{R}$ is an uncertainty measure if it is concave and continuous over $\mathcal{P}$.

We postpone a full justification of the above definition to Section 7 . For the time being, we can explain intuitively the role of concavity as follows. Suppose the secret is generated according to either a distribution $p$ or to another distribution $q$, the choice depending on a coin toss, with head's probability $\lambda$. The coin toss introduces extra randomness in the generation process. Therefore, the overall uncertainty of the adversary about the secret, $U(\lambda \cdot p+(1-\lambda) \cdot q)$, should be no less than the average uncertainty of the two original generation processes considered separately, that is $\lambda U(p)+(1-\lambda) U(q)$. As a matter of fact, most uncertainty measures in QIF do satisfy concavity. Continuity is a technical requirement that comes into play only in Theorem 5.5.

Example 2.3. The following entropy functions, and variations thereof, are often considered in the quantitative security literature as measures of the difficulty or effort necessary for a passive adversary to identify a secret $X$, where $X$ is a random variable over $\mathcal{X}$ distributed according to a known distribution $p(\cdot)$. All of these functions are easily proven to be uncertainty measures in our sense:

- Shannon entropy: $H(p) \triangleq-\sum_{x \in \mathcal{X}} p(x) \log p(x)$, with $0 \log 0=0$ and $\log$ in base 2;

- Error probability entropy: $E(p) \triangleq 1-\max _{x \in \mathcal{X}} p(x)$;

- Guessing entropy: $G(p) \triangleq \sum_{i=1}^{n-1} i \cdot p\left(x_{i}\right)$ with $p\left(x_{1}\right) \geq p\left(x_{2}\right) \geq \ldots \geq p\left(x_{n}\right)$.

Example 2.4. For a somewhat different example of uncertainty, suppose that $\mathcal{X} \subseteq \mathbb{R}$. Then each probability distribution over $\mathcal{X}$ corresponds to a real valued r.v., and we can set $U(X) \triangleq \operatorname{var}(X)$, where $\operatorname{var}(X) \triangleq E\left[(X-\mu)^{2}\right]$, with $\mu=E[X]$, is the familiar variance. This makes intuitive sense, as the higher the variability of $X$, the higher the uncertainty about its value. Let us check that $\operatorname{var}(X)$ is concave and continuous.

Indeed, continuity follows immediately from the definition. Concerning concavity, first note that, for any real $z, E\left[(X-z)^{2}\right]=\operatorname{var}(X)+(\mu-z)^{2}$ (easily checked by writing $(X-z)^{2}$ as $((X-\mu)+(\mu-z))^{2}$, then expanding the square and then applying linearity of expectation.) This implies that $E\left[(X-z)^{2}\right] \geq \operatorname{var}(X)$. Now, let $p, q$ be any two distributions on $\mathcal{X}$, let $\lambda \in[0,1]$ and $r=\lambda \cdot p+(1-\lambda) \cdot q$. Denoting by $\mu_{u}$ and $\operatorname{var}_{u}$, respectively, the expectation and variance of $X$ taken according to a distribution $u$, we have the following.

$$
\begin{aligned}
\operatorname{var}_{r}(X) & =E_{r}\left[\left(X-\mu_{r}\right)^{2}\right] \\
& =\lambda E_{p}\left[\left(X-\mu_{r}\right)^{2}\right]+(1-\lambda) E_{q}\left[\left(X-\mu_{r}\right)^{2}\right] \\
& \geq \lambda \operatorname{var}_{p}(X)+(1-\lambda) \operatorname{var}_{q}(X) .
\end{aligned}
$$

We note that the min-entropy function, $H_{\infty}(p)=-\log \max _{x} p(x)$, is neither concave nor convex, so it does not fit in the present framework. However, one can at least indirectly express min-entropy via the error probability entropy $E(\cdot)$ : $H_{\infty}(p)=-\log (1-E(p))$.

A strategy is a partial function $\sigma: \mathcal{Y}^{*} \rightarrow$ Act such that $\operatorname{dom}(\sigma)$ is non-empty and prefix-closed A strategy is finite if $\operatorname{dom}(\sigma)$ is finite. The length of a finite strategy

\footnotetext{
${ }^{3}$ In fact, concavity does imply continuity except possibly on the frontier of $\mathcal{P}$.

${ }^{4} \mathrm{~A}$ set $B \subseteq \mathcal{Y}^{*}$ is prefix-closed if whenever $\sigma \in B$ and $\sigma^{\prime}$ is a prefix of $\sigma$ then $\sigma^{\prime} \in B$.
} 
is defined as $\max \{|\xi|: \xi \in \operatorname{dom}(\sigma)\}+1$. For each $n \geq 0$ we will let $y^{n}, w^{n}, z^{n}, \ldots$ range over sequences in $\mathcal{Y}^{n}$; given $y^{n}=\left(y_{1}, \ldots, y_{n}\right)$ and $0 \leq j \leq n$, we will let $y^{j}$ denote the first $j$ components of $y^{n},\left(y_{1}, \ldots, y_{j}\right)$. Given a strategy $\sigma$ and an integer $n \geq 0$, the truncation of $\sigma$ at level $n$, denoted as $\sigma \backslash n$, is the finite strategy $\sigma_{\mid \cup_{0 \leq i \leq n}} \mathcal{Y}^{i}$. A finite strategy of length $l$ is complete if $\operatorname{dom}(\sigma)=$ $\cup_{0 \leq i \leq l-1} \mathcal{Y}^{i}$. A strategy $\sigma$ is non-adaptive if whenever $y^{n}$ and $w^{n}$ are two sequences of the same length then $\sigma\left(y^{n}\right)=\sigma\left(w^{n}\right)$ (that is, the decision of which action to play next only depends on the number of past actions); note that finite non-adaptive strategies are necessarily complete. We note that strategies can be described as trees, with nodes labelled by actions and arc labelled by observations, in the obvious way. Any non-adaptive stra-

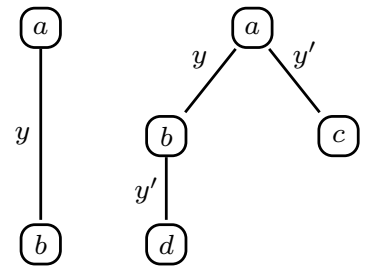

Figure 1. Two strategy trees. tegy also enjoys a simpler representation as a finite or infinite list of actions: we write $\sigma=\left[a_{1}, \ldots, a_{i}, \ldots\right]$ if $\sigma\left(y^{i-1}\right)=a_{i}$, for $i=1,2, \ldots$

Example 2.5. Strategies $\sigma=[\varepsilon \mapsto a, y \mapsto b]$ and $\sigma^{\prime}=\left[\varepsilon \mapsto a, y \mapsto b, y^{\prime} \mapsto c, y y^{\prime} \mapsto d\right]$ can be represented as in Figure 1. Note that the tree's height is one less than the strategy's length.

Remark 2.6. It is worthwhile to comment on two possible objections to our strategy model. First, we do not consider mixed strategies, that is strategies where the next action is chosen probabilistically, rather than deterministically like in our case. It is true that mixed strategies play a key role in Game Theory: equilibria typically arise in the form of profiles of mixed strategies, as in many games any pure (deterministic) strategy could be easily beaten by an opponent. We believe, however, that mixed strategies are irrelevant in the present context: there is only one player here (the adversary), and consequently no meaningful notion of equilibrium. In particular, there is no such role as a defender whose moves depend on the adversary's ones. In game-theoretical terms, the adversary is playing against the Nature, represented by the mechanism.

Another possible objection is that in our model the next action depends solely on the sequence of past observations, rather than on the whole history comprising also the past actions played by the adversary. This limitation can be easily overcome by considering a modified action-based mechanism, where actions are part of the observation: that is, in the new mechanism, the set of observations is $A c t \times \mathcal{Y}$, and one poses $p_{a}((b, y) \mid x) \triangleq p_{a}(y \mid x)$ if $a=b$ and $p_{a}((b, y) \mid x) \triangleq 0$ otherwise, where $p_{a}(\cdot \mid \cdot)$ is the $a$-stochastic matrix of the original mechanism. This way, strategies for the new mechanism automatically take into account the whole history.

2.2. A probability space. Informally, we consider an adversary who repeatedly queries a mechanism, according to a predefined finite strategy. At some point, the strategy will terminate, and the adversary will have collected a sequence of observations $y^{n}=\left(y_{1}, \ldots, y_{n}\right)$. Note that both the length $n$ and the probability of the individual observations $y_{i}$, hence of the whole $y^{n}$, will in general depend both on $X$ and on the strategy played by the adversary. In other words, the distribution $p(\cdot)$ of $X$ and the strategy $\sigma$ together induce a probability distribution on a subset of all observation sequences: the ones that may arise as a result of a complete interaction with the mechanism, according to the played strategy. 
Formally, let $p(\cdot)$ be any given probability distribution over $\mathcal{X}$, which we will often refer to as the prior. For each finite strategy $\sigma$, we define a joint probability distribution $p_{\sigma}(\cdot)$ on $\mathcal{X} \times \mathcal{Y}^{*}$, depending on $\sigma$ and on $p(\cdot)$, as follows. We let $p_{\sigma}(x, \varepsilon) \triangleq 0$ and, for each $j \geq 0$ :

$$
p_{\sigma}\left(x, y_{1}, \ldots, y_{j}, y_{j+1}\right) \triangleq \begin{cases}p(x) \cdot p_{a_{1}}\left(y_{1} \mid x\right) \cdots p_{a_{j}}\left(y_{j} \mid x\right) p_{a_{j+1}}\left(y_{j+1} \mid x\right) \\ \text { if } y^{j} \in \operatorname{dom}(\sigma), y^{j} y_{j+1} \notin \operatorname{dom}(\sigma) \\ 0 & \text { otherwise }\end{cases}
$$

where in the first case $a_{i}=\sigma\left(y^{i-1}\right)$ for $i=1, \ldots, j+1$. In case $\sigma=[a]$, a single action strategy, we will often abbreviate $p_{[a]}(\cdot)$ as $p_{a}(\cdot)$. Note that the support of $p_{\sigma}(\cdot)$ is finite, in particular $\operatorname{supp}\left(p_{\sigma}\right) \subseteq \mathcal{X} \times\left\{y^{j} y: j \geq 0, y^{j} \in \operatorname{dom}(\sigma), y^{j} y \notin \operatorname{dom}(\sigma)\right\}$.

Let $(X, Y)$ be a pair of random variables with outcomes in $\mathcal{X} \times \mathcal{Y}^{*}$, jointly distributed according to $p_{\sigma}(\cdot)$ : here $X$ represents the secret and $Y$ represents the sequence of observations obtained upon termination of the strategy. We shall often use such shortened notations as: $p_{\sigma}\left(x \mid y^{n}\right)$ for $\operatorname{Pr}\left(X=x \mid Y=y^{n}\right), p_{\sigma}\left(y^{n}\right)$ for $\operatorname{Pr}\left(Y=y^{n}\right)$, and so on. Explicit formulas for computing these quantities can be easily derived from the definition of $p_{\sigma}(\cdot)$ and using Bayes rule. We will normally keep the dependence of $(X, Y)$ on $p(\cdot)$ and $\sigma$ implicit. When we want to stress that we are considering $Y$ according to the distribution induced by a specific $\sigma$ (e.g. because different strategies are being considered at the same time), we will write it as $Y_{\sigma}$.

Consider a prior $p(\cdot)$ and a finite strategy $\sigma$, and the corresponding pair of random variables (r.v.) $(X, Y)$. We define the following quantities, expressing average uncertainty, conditional uncertainty and information gain about $X$, that may result from interaction according to strategy $\sigma$ (by convention, below we let $y^{n}$ range over sequences with $p_{\sigma}\left(y^{n}\right)>$ $0)$ :

$$
\begin{aligned}
U(X) & \triangleq U(p) \\
U_{\sigma}(X \mid Y) & \triangleq \sum_{y^{n}} p_{\sigma}\left(y^{n}\right) U\left(p_{\sigma}\left(\cdot \mid y^{n}\right)\right) \\
I_{\sigma}(X ; Y) & \triangleq U(X)-U_{\sigma}(X \mid Y) .
\end{aligned}
$$

Again, we may drop the subscript $\sigma$ from $U_{\sigma}$ and $I_{\sigma}$ if the strategy $\sigma$ is clear from the context. Note that, in the case of Shannon entropy, $I_{\sigma}(X ; Y)$ coincides with the familiar mutual information, traditionally measured in bits. In the case of error entropy, $I_{\sigma}(X ; Y)$ is what is called additive leakage in e.g. [11] and advantage in the cryptographic literature, see e.g. [20] and references therein.

In the rest of the paper, unless otherwise stated, we let $U(\cdot)$ be an arbitrary uncertainty function. The following fact about $I_{\sigma}(X ; Y)$ follows from $U(\cdot)$ 's concavity and Jensen's inequality, plus routine calculations on probability distributions (see Appendix).

Lemma 2.7. $I_{\sigma}(X ; Y) \geq 0$. Moreover $I_{\sigma}(X ; Y)=0$ if $X$ and $Y$ are independent.

Given the definitions in (2.2), adaptive QIF can be defined quite simply. 
Definition 2.8 (QIF under adaptive adversaries). Let $\mathcal{S}$ be a mechanism and $p(\cdot)$ be a prior over $\mathcal{X}$.

(1) For a finite strategy $\sigma$, let $I_{\sigma}(\mathcal{S}, p) \triangleq I_{\sigma}(X ; Y)$.

(2) For an infinite strategy $\sigma$, let $I_{\sigma}(\mathcal{S}, p) \triangleq \lim _{l \rightarrow \infty} I_{\sigma \backslash l}(\mathcal{S}, p)$.

(3) (Maximum IF under $p(\cdot)) I_{\star}(\mathcal{S}, p) \triangleq \sup _{\sigma} I_{\sigma}(\mathcal{S}, p)$.

Note that $l^{\prime} \geq l$ implies $I_{\sigma \backslash l^{\prime}}(\mathcal{S}, p) \geq I_{\sigma \backslash l}(\mathcal{S}, p)$, hence the limit in (2) always exists. Taking the distribution that achieves the maximum leakage, we can define an analog of channel capacity.

Definition 2.9 (Adaptive secrecy capacity). $C(\mathcal{S}) \triangleq \sup _{p \in \mathcal{P}} I_{\star}(\mathcal{S}, p)$.

2.3. Attack Trees. It is sometimes useful to work with a pictorial representation of the adversary's attack steps, under a given strategy and prior. This can take the form of a tree, where each node represents an adversary's belief about the secret, that is, a probability distribution over $\mathcal{X}$. The tree describes the possible evolutions of the belief, depending on the strategy and on the observations. We formally introduce such a representation below: it will be extensively used in the examples. Note that attack trees are different from strategy trees.

A history is a sequence $h \in(A c t \times \mathcal{Y})^{*}$. Let $h=\left(a_{1}, y_{1}, \ldots, a_{n}, y_{n}\right)$ be such a history. Given a prior $p(\cdot)$, we define the update of $p(\cdot)$ after $h$, denoted by $p^{h}(\cdot)$, as the distribution on $\mathcal{X}$ defined by

$$
p^{h}(x) \triangleq p_{\sigma_{h}}\left(x \mid y^{n}\right)
$$

where $\sigma_{h}=\left[a_{1}, \ldots, a_{n}\right]$, provided $p_{\sigma_{h}}\left(y^{n}\right)>0$; otherwise $p^{h}(\cdot)$ is undefined.

The attack tree induced by a strategy $\sigma$ and a prior $p(\cdot)$ is a tree with nodes labelled by probability distributions over $\mathcal{X}$ and arcs labelled with pairs $(y, \lambda)$ of an observation $y$ and a probability value $\lambda$. This tree is obtained from the strategy tree of $\sigma$ as follows. First, note that, in a strategy tree, each node can be identified with the unique history from the root leading to it. Given the strategy tree for $\sigma$ : (a) for each $y \in \mathcal{Y}$ and each node missing an outgoing $y$-labelled arc, attach a new $y$-labelled arc leading to a new node; (b) label each node of the resulting tree by $p^{h}(\cdot)$, where $h$ is the history identifying the node, if $p^{h}(\cdot)$ is defined, otherwise remove the node and its descendants, as well as the incoming arc; (c) label each arc from a node $h$ to a child, represented by a history $h \cdot a \cdot y$, in the resulting tree with $\lambda=p_{a}^{h}(y)$ - to be parsed as $\left(p^{h}\right)_{[a]}(y)$. This is the probability of observing $y$ under a prior $p^{h}(\cdot)$ when submitting action $a$.

The concept of attack trees is demonstrated by a few examples in the next section. Here, we just note the following easy to check facts. For each leaf $h$ of the attack tree: (i) the leaf's label is $p^{h}(\cdot)=p_{\sigma}\left(\cdot \mid y^{n}\right)$, where $y^{n}$ is the sequence of observations in $h$; (ii) if we let $\pi_{h}$ be the product of the probabilities on the edges from the root to the leaf, then $\pi_{h}=p_{\sigma}\left(y^{n}\right)$. Moreover, (iii) each $y^{n}$ s.t. $p_{\sigma}\left(y^{n}\right)>0$ is found in the tree. As a consequence, for a finite strategy, taking (2.2) into account, the uncertainty of $X$ given $Y$ can be computed from the attack tree as:

$$
U_{\sigma}(X \mid Y)=\sum_{h \text { is a leaf }} \pi_{h} U\left(p^{h}\right)
$$




\begin{tabular}{|c|c|c|c|c|}
\hline id & ZIP & Age & Date & Disease \\
\hline 1 & $z_{1}$ & 65 & $d_{2}$ & Heart disease \\
\hline 2 & $z_{1}$ & 65 & $d_{2}$ & Flu \\
\hline 3 & $z_{1}$ & 67 & $d_{2}$ & Short breath \\
\hline 4 & $z_{1}$ & 68 & $d_{1}$ & Obesity \\
\hline 5 & $z_{1}$ & 68 & $d_{1}$ & Heart disease \\
\hline 6 & $z_{3}$ & 66 & $d_{2}$ & Heart disease \\
\hline 7 & $z_{3}$ & 67 & $d_{2}$ & Obesity \\
\hline 8 & $z_{3}$ & 31 & $d_{2}$ & Short breath \\
\hline 9 & $z_{2}$ & 30 & $d_{3}$ & Heart disease \\
\hline 10 & $z_{2}$ & 31 & $d_{3}$ & Obesity \\
\hline
\end{tabular}

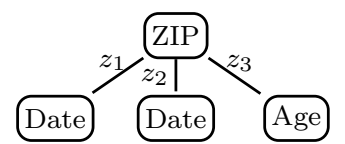

Figure 3. Strategy tree of Example 3.1.

Figure 2. Medical DB of Example 3.1.

\section{EXAMPLES}

We present a few instances of the framework introduced in the previous section. We emphasize that these examples are quite simple and only serve to illustrate our main definitions. In the rest of the paper, we shall use the following notation: we let $\mathrm{u}\left\{x_{1}, \ldots, x_{k}\right\}$ denote the uniform distribution on $\left\{x_{1}, \ldots, x_{k}\right\}$.

Example 3.1 (medical DB). An attacker gets hold of the table shown in Figure 2, which represents a fragment of a hospital's database. Each row of the table contains: a numerical id followed by the ZIP code, age, discharge date and disease of an individual that has been recently hospitalized. The table does not contain personal identifiable information. The attacker gets to know that a certain target individual, John Doe (JD), has been recently hospitalized. However, the attacker is ignorant of the corresponding id in the table and any information about JD, apart from his name. The attacker's task is to identify JD, i.e. to find JD's id in the table, thus learning his disease. The attacker is in a position to ask a source, perhaps the hospital DB, queries concerning non sensitive information (ZIP code, age and discharge date) of any individual, including JD, and compare the answers with the table's entries 5

This situation can be modeled quite simply as an action-based mechanism $\mathcal{S}$, as follows. We pose: $A c t=\{$ ZIP, Age, Date $\} ; \mathcal{X}=\{1, \ldots, 10\}$, the set of possible id's, and $\mathcal{Y}=$ $\mathcal{Y}_{\text {ZIP }} \cup \mathcal{Y}_{\text {Age }} \cup \mathcal{Y}_{\text {Date }}$, where $\mathcal{Y}_{\text {ZIP }}=\left\{z_{1}, z_{2}, z_{3}\right\}, \mathcal{Y}_{\text {Age }}=\{30,31,65,66,67,68\}$ and $\mathcal{Y}_{\text {Date }}=$ $\left\{d_{1}, d_{2}, d_{3}\right\}$. The conditional probability matrices reflect the behaviour of the source when queried about ZIP code, age and discharge date of an individual. We assume that the source is truthful, hence answers will match the entries of the table. For example, $p_{\text {Age }}(y \mid 1)=1$ if $y=65$ and 0 otherwise; $p_{\mathrm{ZIP}}(y \mid 2)=1$ if $y=z_{1}, 0$ otherwise; and so on. Note that this defines a deterministic mechanism. Finally, since the attacker has no clues about JD's id, we set the prior to be the uniform distribution on $\mathcal{X}, p(\cdot)=\mathrm{u}\{1, \ldots, 10\}$.

Assume now that, possibly to protect privacy of individuals, the number of queries to the source about any individual is limited to two. Figure 3 displays a possible attacker's strategy $\sigma$, of length 2. Figure 4 displays the corresponding attack tree, under the given prior. Note that the given strategy is not in any sense optimal. Assume we set $U(\cdot)=H(\cdot)$, Shannon entropy, as a measure of uncertainty. Using (2.4), we can compute $I_{\sigma}(\mathcal{S}, p)=$

\footnotetext{
${ }^{5}$ That this is unsafe is of course well-known from database security: the present example only serves the purpose of illustration.
} 

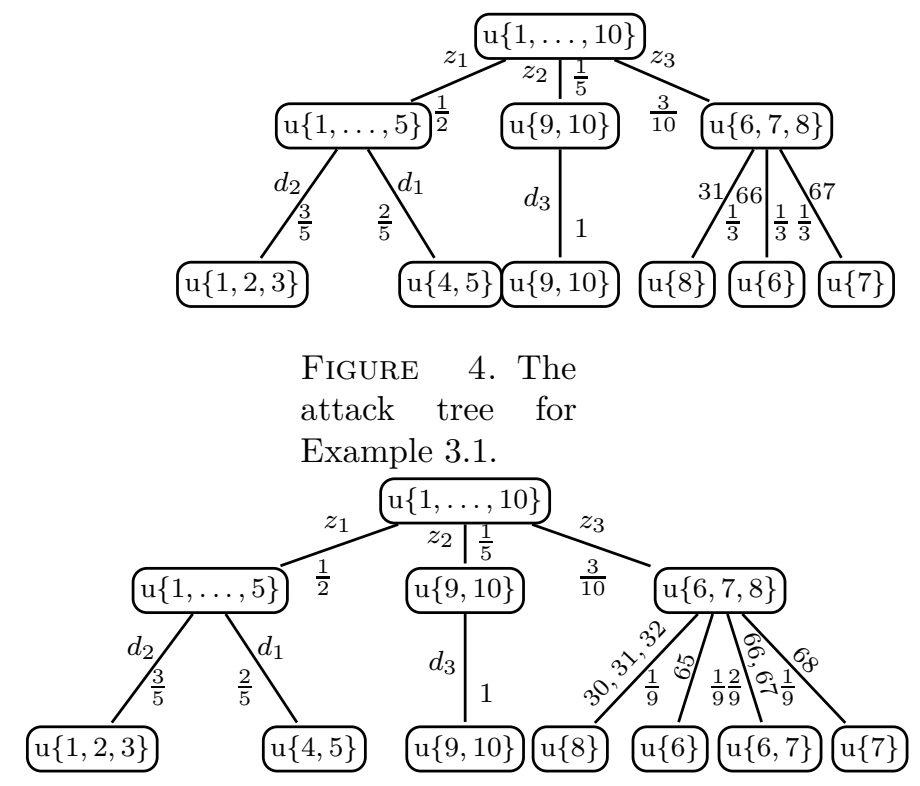

Figure 5. The attack tree for Example 3.2. Leaves with the same label and their incoming arcs have been coalesced.

$H(X)-H(X \mid Y)=\log 10-\frac{3}{10} \log 3-\frac{2}{5} \approx 2.45$ bits. With $U(\cdot)=E(\cdot)$, the error entropy, we have $I_{\sigma}(\mathcal{S}, p)=E(X)-E(X \mid Y)=0.5$.

Example 3.2 (noisy version). We consider a version of the previous mechanism where the public source queried by the attacker is not entirely truthful. In particular, for security reasons, whenever queried about age of an individual, the source adds a uniformly distributed offset $r \in\{-1,0,+1\}$ to the real answer. The only difference from the previous example is that the conditional probability matrix $p_{\text {Age }}(\cdot \mid \cdot)$ is not deterministic anymore. For example, for $x=1$, we have

$$
p_{\text {Age }}(y \mid 1)= \begin{cases}\frac{1}{3} & \text { if } y \in\{64,65,66\} \\ 0 & \text { otherwise }\end{cases}
$$

(also note that we have to insert 29,32,64 and 69 as possible mechanism's observations into $\left.\mathcal{Y}_{\text {Age }}.\right)$ Figure 5 shows the attack tree induced by the strategy $\sigma$ of Figure 3 and the uniform prior in this case. If $U(\cdot)=H(\cdot)$ we obtain $I_{\sigma}(\mathcal{S}, p)=\log 10-\frac{3}{10} \log 3-\frac{8}{15} \approx 2.31$ bits; if $U(\cdot)=E(\cdot)$, instead, $I_{\sigma}(\mathcal{S}, p)=\frac{13}{30} \approx 0.43$.

Example 3.3 (cryptographic devices). We can abstractly model a cryptographic device as a function $f$ taking pairs of a key and a message into observations, thus, $f: \mathcal{K} \times \mathcal{M} \rightarrow \mathcal{Y}$. Assume the attacker can choose the message $m \in \mathcal{M}$ fed to the device, while the private key $k$ is fixed and unknown to him. This clearly yields an action-based mechanism $\mathcal{S}$ where $\mathcal{X}=\mathcal{K}, A c t=\mathcal{M}$ and $\mathcal{Y}$ are the observations. If we assume the observations noiseless, then the conditional probability matrices are defined by

$$
p_{m}(y \mid k)=1 \text { iff } f(k, m)=y .
$$


We obtain therefore a deterministic mechanism. This is the way, for example, modular exponentiation is modeled in [26]. More realistically, the observations will be noisy, due e.g. to the presence of "algorithmic noise". For example, assume $\mathcal{Y} \subseteq \mathbb{N}$ is the set of possible Hamming weights of the ciphertexts (this is related to power analysis attacks, see e.g. [25].) Then we may set

$$
p_{m}(y \mid k)=\operatorname{Pr}(f(k, m)+N=y)
$$

where $N$ is a random variable modelling noise. For example, in the model of DES S-Boxes considered in [7], $\mathcal{K}=\mathcal{M}=\{0,1\}^{6}$, while $\mathcal{Y}=\{0,1,2, \ldots\}$ is the set of observations: the (noisy) Hamming weight of the outputs of the target S-Box. In this case, $N$ is taken to be the cumulative weight of the seven S-Boxes other than the target one. It is sensible to assume this noise to be binomially distributed: $N \sim B(m, p)$, with $m=28$ and $p=\frac{1}{2}$. See [7] for details.

\section{Comparing Adaptive and Non-adaptive Strategies}

Conceptually, we can broadly classify mechanisms into two categories, depending on the size of the set Act. The first category consists of systems with a huge - exponential, in the size of any reasonable syntactic description - number of actions. The second category consists of systems with an "affordable" number of actions. In the first category, we find, for instance, complex cryptographic hardware, possibly described via boolean circuits or other "succinct" notations (cf. the public key exponentiation algorithms considered in [26].) In the second category, we find systems explicitly described by tables, such as databases (Example 3.1 and 3.2) and S-Boxes (Example 3.3.) It makes sense to assess the difficulty of analysing the security of mechanisms separately for these two categories.

4.1. Systems in Succinct Form. We argue that the analysis of such systems is in general an intractable problem, even if restricted to simple special instances of the non-adaptive case. We consider the problem of deciding if there is a finite strategy over a given time horizon yielding an information flow exceeding a given threshold. This decision problem is of course simpler than the problem of finding an optimal strategy over a finite time horizon: indeed, any algorithm for finding the optimal strategy can also be used to answer the first problem. We give some definitions.

Definition 4.1 (Systems in boolean forms). Let $t, u, v$ be nonnegative integers. We say a mechanism $\mathcal{S}=\left(\mathcal{X}, \mathcal{Y}\right.$, Act, $\left.\left\{M_{a}: a \in A c t\right\}\right)$ is in $(t, u, v)$-boolean form if $\mathcal{X}=\{0,1\}^{t}$, Act $=\{0,1\}^{u}, \mathcal{Y}=\{0,1\}^{v}$ and there is a boolean function $f:\{0,1\}^{t+u} \rightarrow\{0,1\}^{v}$ such that for each $x \in \mathcal{X}, y \in \mathcal{Y}$ and $a \in A c t, p_{a}(y \mid x)=1$ iff $f(x, a)=y$. The size of $\mathcal{S}$ is defined as the syntactic size of the smallest boolean formula for $f$.

It is not difficult to see that the class of boolean forms coincides, up to suitable encodings, with that of deterministic systems.

Definition 4.2 (Adaptive Bounding Problem in succinct form, ABPS). Given a mechanism $\mathcal{S}$ in a $(t, u, v)$-boolean form, represented by a boolean expression, a prior distribution $p(\cdot)$, $l \geq 1$ and $T \geq 0$, decide if there is a strategy $\sigma$ of length $\leq l$ such that $I_{\sigma}(\mathcal{S} ; p)>T$. 
In the following theorem, we shall assume, for simplicity, the following reasonable properties of $U(\cdot)$ : if $p(\cdot)$ concentrates all the probability mass on a single element, and $q(\cdot)$ is the uniform distribution, then $0=U(p)<U(q)$. A slight modification of the argument also works without this assumption. The theorem says that even length 1 (hence non-adaptive) strategies are difficult to assess.

Theorem 4.3. Assume $U(\cdot)$ satisfies the above stated property. Then the ABPS is NP-hard, even if fixing $t=v=l=1$, and $T=0$.

Proof. We reduce from the satisfiability problem for boolean formulae. Let $\phi\left(z_{1}, \ldots, z_{u}\right)=$ $\phi(\tilde{z})$ be an arbitrary boolean formula with $u$ free boolean variables $z_{1}, \ldots, z_{u}$. We show how to build in polynomial time out of $\phi(\tilde{z})$ a mechanism $\mathcal{S}$ in $(1, u, 1)$-boolean form, and a prior $p(\cdot)$, with the following property: there is a length 1 strategy $\sigma$ s.t. $I_{\sigma}(\mathcal{S}, p)>0$ iff $\phi(\tilde{z})$ is satisfiable. Take $\mathcal{X}=\mathcal{Y}=\{0,1\}$ and $A c t=\{0,1\}^{u}$. Let the mechanism $\mathcal{S}$ be defined by the boolean function $f\left(x, z_{1}, \ldots, z_{u}\right)=x \wedge \phi(\tilde{z})$. Let $p(\cdot)$ be the uniform prior on $\mathcal{X}=\{0,1\}$. Now, if there is an action $\tilde{b}=\left(b_{1}, \ldots, b_{u}\right) \in A c t$ such that $\phi(\tilde{b})=1(\phi(\tilde{z})$ is satisfiable $)$ then clearly we will have that $Y=X \wedge \phi(\tilde{b})$ is logically equivalent to $X$, hence $U(X \mid Y)=0$. Consequently, setting $\sigma=[\varepsilon \mapsto \tilde{b}]$, we will have that $I_{\sigma}(\mathcal{S}, p)=U(X)-U(X \mid Y)>0$. On the other hand, if $\phi(\tilde{z})$ is not satisfiable, then for any $\tilde{b} \in$ Act we will have that $Y=X \wedge \phi(\tilde{b})$ is logically equivalent to 0 , hence $U(X \mid Y)=U(X)$. Consequently, for any $\sigma=[\varepsilon \mapsto \tilde{b}]$, we will have $I_{\sigma}(\mathcal{S}, p)=U(X)-U(X \mid Y)=0$.

We should stress again that the above result concerns the difficulty of analyzing succinct mechanisms under the simplest possible form of attacker; by no means it entails that the adaptive and non adaptive attackers are equally effective. The following example should clarify that between the two forms of attacker, there can be a huge difference in terms of effectiveness.

Example 4.4 (envelopes). A secret bit $s \in\left\{b_{0}, b_{1}\right\}$ and a numbered envelope $e \in\{1, \ldots, N\}$ are drawn according to some distribution $p$. A piece of paper with the value of $s$ written on it is put into $e$. All other envelopes are filled with a piece of paper revealing the envelope containing the secret, that is $e$. The adversary can choose and open any of the envelopes and examine its content: that is, actions of this mechanism are envelope numbers.

Assume the envelope is chosen uniformly at random. Clearly, the obvious adaptive strategy leads the adversary to discover the secret after at most two actions. On the other hand, a non-adaptive, brute-force strategy will lead him to examine one envelope after the other, ignoring the suggestion given in the envelopes opened so far, leading to a strategy of length $N-1$.

4.2. General Systems. The following results apply in general, but they are particularly significative for systems with a moderate number of actions. The next theorem essentially says that, up to an expansion factor bounded by $|A c t|$, non-adaptive strategies are as efficient as adaptive ones. In fact, given any strategy $\sigma$, one can construct a non-adaptive $\sigma^{\prime}$ that is only moderately larger than $\sigma$ and achieves at least the same leakage, as follows. In any history induced by $\sigma$, each action can occur at most $l$ times, where $l$ is the length of $\sigma$, and the order in which different actions appear in the history is not relevant as to the final belief that is obtained. For any history of $\sigma$ to be simulated by an history of $\sigma^{\prime}$, it is therefore enough that the latter offers all the distinct actions offered by $\sigma$, each repeated $l$ times. Note that, for a strategy $\sigma$, the number of distinct actions that appear in $\sigma$ is $|\operatorname{range}(\sigma)|$. 
Theorem 4.5. For each finite strategy $\sigma$ of length $l$ it is possible to build a non-adaptive finite strategy $\sigma^{\prime}$ of length $\mid$ range $(\sigma) \mid \times l$, such that $I_{\sigma^{\prime}}(\mathcal{S}, p) \geq I_{\sigma}(\mathcal{S}, p)$.

Proof. Let range $(\sigma)=\left\{a_{1}, \ldots, a_{h}\right\}$ and let $\sigma^{\prime}$ be any non-adaptive strategy that plays each of $a_{1}, \ldots, a_{h}$ for $l$ times, for example, $\sigma^{\prime}=\left[a_{1}, \ldots, a_{h}, \ldots, a_{1}, \ldots, a_{h}\right]$ ( $l$ times); note that the length of $\sigma^{\prime}$ is $h \times l$, as required. For any $y^{j}(j \leq l)$, we shall denote by $\sigma^{\prime}-y^{j}$ the non-adaptive strategy of length $h \times l-j$ obtained by removing from $\sigma^{\prime}$, seen as a list, $j$ actions $b_{1}, \ldots, b_{j}$, where $b_{1}=\sigma(\varepsilon), \ldots, b_{j}=\sigma\left(y^{j-1}\right)$.

Denote by $Y_{\sigma}$ and $Y_{\sigma^{\prime}}$ the r.v. on $\mathcal{Y}^{*}$ corresponding to $\sigma$ and $\sigma^{\prime}$, respectively. We will show that $U\left(X \mid Y_{\sigma}\right) \geq U\left(X \mid Y_{\sigma^{\prime}}\right)$. Take any $x \in \mathcal{X}$ s.t. $p(x)>0$ and $y^{j} \in \operatorname{dom}\left(p_{\sigma}\right)$. We note that, for any sequence $y^{h l-j}$, and for an appropriate interleaving of the two sequences $y^{h l-j}$ and $y^{j}$ that here we denote by just $y^{h l-j}, y^{j}$, we have that

$$
p_{\sigma^{\prime}-y^{j}}\left(y^{h l-j} \mid x\right) p_{\sigma}\left(y^{j} \mid x\right)=p_{\sigma^{\prime}}\left(y^{h l-j}, y^{j} \mid x\right) .
$$

From (4.1), it follows that

$$
p_{\sigma}\left(y^{j} \mid x\right)=\sum_{y^{h l-j}} p_{\sigma^{\prime}-y^{j}}\left(y^{h l-j} \mid x\right) p_{\sigma}\left(y^{j} \mid x\right)=\sum_{y^{h l-j}} p_{\sigma^{\prime}}\left(y^{h l-j}, y^{j} \mid x\right) .
$$

Now, for any $x$ and $y^{j}$ such that $p(x)>0$ and $p_{\sigma}\left(y^{j}\right)>0$, we have the following.

$$
\begin{aligned}
p_{\sigma}\left(x \mid y^{j}\right) & =\frac{p_{\sigma}\left(y^{j} \mid x\right) p(x)}{p_{\sigma}\left(y^{j}\right)} \\
& =\sum_{y^{h l-j}} p_{\sigma^{\prime}}\left(y^{h l-j}, y^{j} \mid x\right) \frac{p(x)}{p_{\sigma}\left(y^{j}\right)} \\
& =\sum_{y^{h l-j}} \frac{p_{\sigma^{\prime}}\left(x \mid y^{h l-j}, y^{j}\right) p_{\sigma^{\prime}}\left(y^{h l-j}, y^{j}\right)}{p(x)} \frac{p(x)}{p_{\sigma}\left(y^{j}\right)} \\
& =\sum_{y^{h l-j}} p_{\sigma^{\prime}}\left(x \mid y^{h l-j}, y^{j}\right) \frac{p_{\sigma^{\prime}}\left(y^{h l-j}, y^{j}\right)}{p_{\sigma}\left(y^{j}\right)}
\end{aligned}
$$

where in the second equality of (4.3) we have applied (4.2). It is an easy matter to show that $\sum_{y^{h l-j}} \frac{p_{\sigma^{\prime}}\left(y^{h l-j}, y^{j}\right)}{p_{\sigma}\left(y^{j}\right)}=1$ (this is basically a consequence of (4.1); we leave the details to the interested reader.) Thus (4.4) shows that $p_{\sigma}\left(\cdot \mid y^{j}\right)$ can be expressed as a convex combination of the distributions $p_{\sigma^{\prime}}\left(\cdot \mid y^{h l-j}, y^{j}\right)$, for $y^{h l-j} \in \mathcal{Y}^{h l-j}$. Using this fact, the concavity of $U(\cdot)$ and Jensen's inequality, we arrive at the following.

$$
U\left(p_{\sigma}\left(\cdot \mid y^{j}\right)\right) \geq \sum_{y^{h l-j}} U\left(p_{\sigma^{\prime}}\left(\cdot \mid y^{h l-j}, y^{j}\right)\right) \frac{p_{\sigma^{\prime}}\left(y^{h l-j}, y^{j}\right)}{p_{\sigma}\left(y^{j}\right)}
$$


We finally can compute the following lower-bound for $U\left(X \mid Y_{\sigma}\right)$.

$$
\begin{aligned}
U\left(X \mid Y_{\sigma}\right) & =\sum_{y^{j}} p_{\sigma}\left(y^{j}\right) U\left(p_{\sigma}\left(\cdot \mid y^{j}\right)\right) \\
& \geq \sum_{y^{j}} p_{\sigma}\left(y^{j}\right) \sum_{y^{h l-j}} \frac{p_{\sigma^{\prime}}\left(y^{h l-j}, y^{j}\right)}{p_{\sigma}\left(y^{j}\right)} U\left(p_{\sigma^{\prime}}\left(\cdot \mid y^{h l-j}, y^{j}\right)\right) \\
& =\sum_{y^{j}} \sum_{y^{h l-j}} p_{\sigma^{\prime}}\left(y^{h l-j}, y^{j}\right) U\left(p_{\sigma^{\prime}}\left(\cdot \mid y^{h l-j}, y^{j}\right)\right) \\
& =\sum_{y^{h l}} p_{\sigma^{\prime}}\left(y^{h l}\right) U\left(p_{\sigma^{\prime}}\left(\cdot \mid y^{h l}\right)\right)=U\left(X \mid Y_{\sigma^{\prime}}\right)
\end{aligned}
$$

where the inequality (4.6) follows from (4.5).

In deterministic systems, repetitions of the same action are not relevant: this leads to the following improved upper bound on the length of the non-adaptive $\sigma^{\prime}$ that simulates $\sigma$.

Proposition 4.6. If the mechanism $\mathcal{S}$ is deterministic, then the upper-bound in the previous theorem can be simplified to $|\operatorname{range}(\sigma)|$.

Proof. Let $\sigma$ be any finite non-adaptive strategy for $\mathcal{S}$. Suppose there is an action $a$ that occurs at least twice in $\sigma$, seen as a tuple of actions, and let $\sigma_{-}$be the non-adaptive strategy obtained by removing the first occurrence of $a$ from $\sigma$, seen as a list. Assume the two $a$ 's occur at position $i$ and $j, i<j$, of $\sigma$. Since $\mathcal{S}$ is deterministic, it is easily seen that, for each $y^{n}=\left(y_{1}, \ldots, y_{n}\right)$, if $y_{i} \neq y_{j}$ then for each $x p_{\sigma}\left(y^{n} \mid x\right)=0$ (as submitting twice the same action $a$ cannot give rise to two different answers $y_{i}$ and $y_{j}$ ), and as a consequence $p_{\sigma}\left(y^{n}\right)=0$. On the other hand, if $y_{i}=y_{j}$, then, denoting by $y^{n-1}$ the sequence obtained by removing $y_{i}$ from $y^{n}$, for each $x$ we have: $p_{\sigma}\left(y^{n} \mid x\right)=p_{\sigma_{-}}\left(y^{n-1} \mid x\right)\left(\right.$ as $p\left(y_{i} \mid x\right)=p\left(y_{j} \mid x\right)$ is either 0 or 1$)$, and as a consequence $p_{\sigma}\left(y^{n}\right)=p_{\sigma^{\prime}}\left(y^{n-1}\right)$ and $p_{\sigma}\left(x \mid y^{n}\right)=p_{\sigma_{-}}\left(x \mid y^{n-1}\right)$. This implies that $U\left(X \mid Y_{\sigma}\right)=U\left(X \mid Y_{\sigma_{-}}\right)$. Repeating this elimination step, we can eventually get rid of all the duplicates in $\sigma$, while preserving the value of $I_{\sigma}(\mathcal{S}, p)$. Applying this fact to the strategy $\sigma^{\prime}$ defined in the proof of Theorem 4.5, we can come up with a strategy $\sigma^{\prime \prime}$ of length $|\operatorname{range}(\sigma)|$ such that $I_{\sigma^{\prime \prime}}(\mathcal{S}, p)=I_{\sigma_{-}}(\mathcal{S}, p)$.

Example 4.7. We reconsider Example 3.1. For the adaptive strategy $\sigma$ defined in Figure 3. we have already shown that, for $U(\cdot)=H(\cdot), I_{\sigma}(\mathcal{S}, p) \approx 2.45$. Consider now the nonadaptive strategy $\sigma^{\prime}=$ [ZIP, Date, Age], which is just one action longer than $\sigma$. The corresponding attack tree is reported in Figure 66 the final partition obtained with $\sigma^{\prime}$ is finer than the one obtained with $\sigma$. In fact, $I_{\sigma^{\prime}}(\mathcal{S}, p)=\log 10-\frac{2}{5} \approx 2.92>I_{\sigma}(\mathcal{S}, p)$.

The results discussed above are important from the point of view of the analysis of randomization mechanisms. They entail that, for systems with a moderate number of actions, analyzing adaptive strategies is essentially equivalent to analyzing non-adaptive ones. The latter task can be much easier to accomplish. For example, results on asymptotic rate of convergence of non-adaptive strategies are available (e.g. [7, Th. IV.3].) They permit to analytically assess the resistance of a mechanism as the length of the considered strategies grows. 


\section{Maximum Leakage}

In this section we show that the class of adaptive and non adaptive strategies induce the same maximum leakage, where the maximum is taken over all strategies. For truly probabilistic mechanisms, strategies achieving maximum leakage are in general infinite. A key notion is that of indistinguishability: an equivalence relation over $\mathcal{X}$ s.t. $x$ and $x^{\prime}$ are indistinguishable if, no matter what strategy the adversary will play, he cannot tell them apart.

Definition 5.1 (Indistinguishability). We define the following equivalence relation over $\mathcal{X}$ :

$$
x \equiv x^{\prime} \quad \text { iff } \quad \text { for each finite } \sigma: p_{\sigma}(\cdot \mid x)=p_{\sigma}\left(\cdot \mid x^{\prime}\right) .
$$

Despite being based on a universal quantification over all finite strategies, indistinguishability is in fact quite easy to characterize, also computationally. For each $a \in A c t$, consider the equivalence relation defined by $x \equiv_{a} x^{\prime}$ iff $p_{a}(\cdot \mid x)=p_{a}\left(\cdot \mid x^{\prime}\right)$. We have the following result (see the Appendix for a proof.)

Lemma 5.2. $x \equiv x^{\prime}$ iff for each $a \in$ Act, $p_{a}(\cdot \mid x)=p_{a}\left(\cdot \mid x^{\prime}\right)$. In other words, $\equiv$ is $\cap_{a \in A c t} \equiv_{a}$.

Now, consider $\mathcal{X} / \equiv$, the set of equivalence classes of $\equiv$, and let $c$ ranges over this set. Let $[X]$ be the r.v. whose outcome is the equivalence class of $X$ according to $\equiv$. Note that $p(c) \triangleq \operatorname{Pr}([X]=c)=\sum_{x \in c} p(x)$. We consistently extend our $I$-notation by defining

$$
U(X \mid[X]) \triangleq \sum_{c} p(c) U(p(\cdot \mid[X]=c)) \quad \text { and } \quad I(X ;[X]) \triangleq U(X)-U(X \mid[X]) .
$$

More explicitly, $p(\cdot \mid[X]=c)$ denotes the distribution over $\mathcal{X}$ that yields $p(x) / p(c)$ for $x \in c$ and 0 elsewhere; we will often abbreviate $p(\cdot \mid[X]=c)$ just as $p(\cdot \mid c)$. Note that $I(X ;[X])$ expresses the information gain about $X$ when the attacker gets to know the indistinguishability class of the secret. As expected, this is an upper-bound to the information that can be gained by playing any strategy.

Theorem 5.3. $I_{\star}(\mathcal{S}, p) \leq I(X ;[X])$.

Proof. Fix any finite strategy $\sigma$ and prior $p(\cdot)$. It is sufficient to prove that $U(X \mid Y) \geq$ $U(X \mid[X])$. The proof exploits the concavity of $U$. First, we note that, for each $x$ and $y^{j}$ of nonzero probability we have ( $c$ below ranges over $\mathcal{X} / \equiv$ ):

$$
p_{\sigma}\left(x \mid y^{j}\right)=\sum_{c} \frac{p_{\sigma}\left(x, y^{j}, c\right)}{p_{\sigma}\left(y^{j}\right)}=\sum_{c} p_{\sigma}\left(c \mid y^{j}\right) p_{\sigma}\left(x \mid y^{j}, c\right) .
$$

By (5.1), concavity of $U(\cdot)$ and Jensen's inequality

$$
U\left(p\left(\cdot \mid y^{j}\right)\right) \geq \sum_{c} p_{\sigma}\left(c \mid y^{j}\right) U\left(p_{\sigma}\left(\cdot \mid y^{j}, c\right)\right) .
$$


Now, we can compute as follows (as usual, $y^{j}$ below runs over sequences of nonzero probability):

$$
\begin{aligned}
U(X \mid Y) & =\sum_{y^{j}} p_{\sigma}\left(y^{j}\right) U\left(p_{\sigma}\left(\cdot \mid y^{j}\right)\right) \geq \sum_{y^{j}, c} p_{\sigma}\left(y^{j}\right) p_{\sigma}\left(c \mid y^{j}\right) U\left(p_{\sigma}\left(\cdot \mid y^{j}, c\right)\right) \\
& =\sum_{y^{j}, c} p_{\sigma}\left(y^{j}\right) p_{\sigma}\left(c \mid y^{j}\right) U(p(\cdot \mid c))=\sum_{c}\left(\sum_{y^{j}} p_{\sigma}\left(y^{j}, c\right)\right) U(p(\cdot \mid c)) \\
& =\sum_{c} p(c) U(p(\cdot \mid c))=U(X \mid[X])
\end{aligned}
$$

where: (5.3) is justified by (5.2); and the first equality in (5.4) follows from the fact that, for each $x, p_{\sigma}\left(x \mid y^{j}, c\right)=p(x \mid c)$ (once the equivalence class of the secret is known, the observation $y^{j}$ provides no further information about the secret.)

As to the maximal achievable information, we start our discussion from deterministic mechanism.

Proposition 5.4. Let $\mathcal{S}$ be deterministic. Let $\sigma=\left[a_{1}, \ldots, a_{k}\right]$ be a non-adaptive strategy that plays all actions in Act once. Then $I_{\star}(\mathcal{S}, p)=I_{\sigma}(\mathcal{S}, p)$.

Proof. Let $(X, Y) \sim p_{\sigma}(\cdot)$. We prove that $U(X \mid Y)=U(X \mid[X])$. We first note that for each $c \in \mathcal{X} / \equiv$ there is exactly one sequence $y_{c}^{k}$ s.t. $p_{\sigma}\left(y_{c}^{k} \mid c\right)=1$ : this follows from $\mathcal{S}$ being deterministic. Moreover, if $c \neq c^{\prime}$ then $y_{c}^{k} \neq y_{c^{\prime}}^{k}$ : otherwise, it would follow that $p_{a_{i}}(y \mid c)=p_{a_{i}}\left(y \mid c^{\prime}\right)$ for each $a_{i} \in$ Act and $y \in \mathcal{Y}$, contrary to Lemma 5.2 (note that $p(\cdot \mid c$ ) is the same as $p(\cdot \mid x)$, for any $x \in c$.) These facts can be used to show, through easy manipulations, that $p\left(x \mid y_{c}^{k}\right)=p(x \mid c)$ for each $x$. As a consequence, one can compute as follows.

$$
\begin{aligned}
U(X \mid Y) & =\sum_{y^{k}} p_{\sigma}\left(y^{k}\right) U\left(p_{\sigma}\left(\cdot \mid y^{k}\right)\right) \\
& =\sum_{c} p(c) \sum_{y^{k}} p_{\sigma}\left(y^{k} \mid c\right) U\left(p_{\sigma}\left(\cdot \mid y^{k}\right)\right) \\
& =\sum_{c} p(c) U\left(p_{\sigma}\left(\cdot \mid y_{c}^{k}\right)\right) \\
& =\sum_{c} p(c) U\left(p_{\sigma}(\cdot \mid c)\right) \\
& =U(X \mid[X]) .
\end{aligned}
$$

Hence, in the deterministic case, the maximal gain in information is obtained by a trivial brute-force strategy where all actions are played in any fixed order. It is instructive to observe such a strategy at work, under the form of an attack tree. The supports of the distributions that are at the same level constitute a partition of $\mathcal{X}$ : more precisely, the partition at level $i(1 \leq i \leq k)$ is given by the equivalence classes of the relation $\cap_{j=1}^{i} \equiv_{a_{j}}$. An example of this fact is illustrated by the attack tree in Figure 6. relative to the nonadaptive strategy [ZIP, Date, Age] for the mechanism in Example 3.1. This fact had been already observed in [26] for the restricted model considered there. Indeed, one would obtain the model of [26] by stripping the probabilities off the tree in Figure 6.

The general probabilistic case is more complicated. Essentially, any non-adaptive strategy where each action is played infinitely often achieves the maximum information gain. 


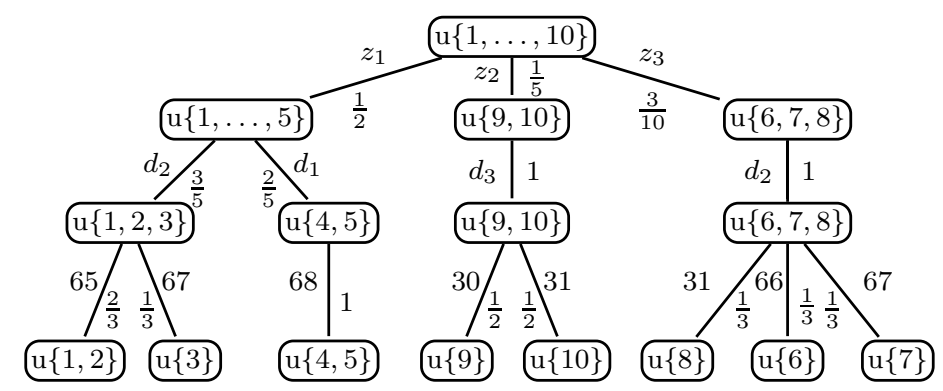

FiguRE 6. The attack tree corresponding to the the non-adaptive strategy [ZIP, Date, Age] for Example [3.1]

The next theorem considers one such strategy; the proof of this result is reported in Appen$\operatorname{dix}$ B.

Theorem 5.5. There is a total, non-adaptive strategy $\sigma$ s.t. $I_{\sigma}(\mathcal{S}, p)=I(X ;[X])$. Consequently, $I_{\star}(\mathcal{S}, p)=I(X ;[X])$.

Of course, as shown in the preceding section, finite adaptive strategies can be more efficient in terms of length by a factor of $|A c t|$ when compared with non-adaptive ones. Concerning capacity, we do not have a general formula for the maximizing distribution. In what follows, we limit our discussion to two important cases for $U(\cdot)$, Shannon entropy and error entropy. In both cases, capacity only depends on the number $K$ of indistinguishability classes. For guessing entropy, we conjecture that $C(\mathcal{S})=\frac{K-1}{2}$, but at the moment a proof of this fact escapes us.

Theorem 5.6. The following formulae holds, where $K=|\mathcal{X}| \equiv \mid$.

- For $U=H$ (Shannon entropy), $C(\mathcal{S})=\log K$.

- For $U=E$ (Error entropy), $C(\mathcal{S})=1-\frac{1}{K}$.

Proof. Let $x_{i}$ any representative of class $c_{i}$, for $i=1, \ldots, K$.

- $U=H$. By the symmetry of mutual information in the case of Shannon entropy, we have

$$
\begin{aligned}
I(X ;[X]) & =H([X])-\underbrace{H([X] \mid X)}_{=0}=H([X]) \\
& =-\sum_{c_{i}} p\left(c_{i}\right) \log p\left(c_{i}\right) \leq \log K
\end{aligned}
$$

where the last inequality follows from the property of Shannon entropy that $H(q) \leq$ $\log |\operatorname{supp}(q)|$, for any distribution $q$. On the other hand, if we take the distribution $p(\cdot)$ defined as $p\left(x_{i}\right)=\frac{1}{K}$, and $p(x)=0$ elsewhere, we can easily compute that $I(X ;[X])=$ $\log K$. 
- $U=E$. Let $p(\cdot)$ be any prior and assume without loss of generality that $p\left(x_{i}\right)=$ $\max _{x \in c_{i}} p(x)$ for each $i$, and furthermore that $p\left(x_{1}\right)=\max _{x} p(x)$. By easy manipulations, we have:

$$
\begin{aligned}
I(X ;[X]) & =E(X)-E(X \mid[X]) \\
& =\left(1-p\left(x_{1}\right)\right)-\left(1-\sum_{c_{i}} p\left(c_{i}\right) \frac{p\left(x_{i}\right)}{p\left(c_{i}\right)}\right) \\
& =\left(\sum_{i=1}^{K} p\left(x_{i}\right)\right)-p\left(x_{1}\right)=\sum_{i=2}^{K} p\left(x_{i}\right) .
\end{aligned}
$$

Now it is easily checked that the last term in this chain is $\leq 1-\frac{1}{K}$ : this is done by separately considering the two cases $\max _{x} p(x)=p\left(x_{1}\right) \leq \frac{1}{K}$ and $\max _{x} p(x)=p\left(x_{1}\right)>\frac{1}{K}$. On the other hand, if we take, as above, the distribution $p(\cdot)$ defined as $p\left(x_{i}\right)=\frac{1}{K}$, and $p(x)=0$ elsewhere, we can easily compute that $I(X ;[X])=1-\frac{1}{K}$.

Example 5.7. Consider the mechanism defined in Example 3.1. One has the following capacities: for $U(\cdot)=H(\cdot), C(\mathcal{S})=\log 8=3$, while for $U(\cdot)=E(\cdot), C(\mathcal{S})=\frac{7}{8}=0.875$.

\section{Computing Optimal Finite Strategies}

We show that, for finite strategies, $I_{\sigma}(\mathcal{S}, p)$ can be expressed recursively as a Bellman equation. This allows for calculation of optimal finite strategies based on standard algorithms, such as backward induction.

6.1. A Bellman Equation. Let us introduce some terminology. For each $y$, the $y$-derivative of $\sigma$, denoted $\sigma_{y}$, is the function defined thus, for each $y^{j} \in \mathcal{Y}^{*}: \sigma_{y}\left(y^{j}\right) \triangleq \sigma\left(y y^{j}\right)$. Note that if $\sigma$ has length $l>1$, then $\sigma_{y}$ is a strategy of height $\leq l-1$. For $l=1, \sigma_{y}$ is the empty function. Recall that according to (2.3) , for $h=a y$, we have 6

$$
p^{a y}(x)=p_{a}(x \mid y) .
$$

By convention, we let $I_{\sigma}(\cdots)$ denote 0 when $\sigma$ is empty. Moreover, we write $I_{[a]}(\cdots)$ as $I_{a}(\cdots)$.

Lemma 6.1. Let $p(\cdot)$ be any prior on $\mathcal{X}$. Let $\sigma$ be a strategy with $\sigma(\varepsilon)=a$. Then $I_{\sigma}(\mathcal{S} ; p)=I_{a}(\mathcal{S} ; p)+\sum_{y} p_{a}(y) I_{\sigma_{y}}\left(\mathcal{S} ; p^{a y}\right)$.

We introduce some additional notation to be used in the proof of this lemma. Let $l$ denote the length of a strategy $\sigma$, and let $(X, Y)$ be distributed according to $p_{\sigma}(\cdot)$. We can decompose $Y$ as the concatenation of the 1st observation and whatever sequence of observations is left, thus: $Y=Y_{1} \cdot Y_{s}$. Here, $Y_{1}$ takes values on $\mathcal{Y}$, while $Y_{s}$ takes values on a subset of $\cup_{1 \leq j \leq l} \mathcal{Y}^{j}$ - in particular, if $l=1, Y_{s}$ takes on the value $\varepsilon$ with probability 1 . In what follows, we denote the marginal distribution of $Y_{1}$ under $\sigma$ just as $p_{\sigma}(y)$, and that of $Y_{s}$ as $p_{\sigma}\left(y^{j}\right)$, for generic $y$ and $y^{j}$.

\footnotetext{
${ }^{6}$ In terms of a given prior $p(\cdot)$ and of the matrices of $\mathcal{S}$, this can be also expressed as: $p^{a y}(x)=$ $\frac{p_{a}(y \mid x) p(x)}{\sum_{x^{\prime}} p_{a}\left(y \mid x^{\prime}\right) p\left(x^{\prime}\right)}$.
} 
Proof. (of Lemma 6.1) It is an easy matter to prove the following equations, for each prior $p(\cdot)$, finite strategy $\sigma$ with $\sigma(\varepsilon)=a$, sequence $y^{j}$, observation $y$, one has (below, $y$ and $y^{j}$ run over elements of nonzero probability; moreover, for any prior $p(\cdot)$, history $h$ and strategy $\sigma$, the term $p_{\sigma}^{h}$ is to be parsed as $\left.\left(p^{h}\right)_{\sigma}\right)$ :

$$
\begin{aligned}
p_{\sigma}(y) & =p_{a}(y) \\
p_{\sigma}(x \mid y) & =p_{a}(x \mid y)=p^{a y}(x) \\
p_{\sigma}\left(x \mid y y^{j}\right) & =p_{\sigma_{y}}^{a y}\left(x \mid y^{j}\right) \\
p_{\sigma}\left(y^{j} \mid y\right) & =p_{\sigma_{y}}^{a y}\left(y^{j}\right) .
\end{aligned}
$$

By applying equalities (6.1), (6.2), (6.3) and (6.4) above as appropriate, we have:

$$
\begin{aligned}
I_{\sigma}(\mathcal{S}, p) & =I(X ; Y) \\
& =\left[U(X)-U\left(X \mid Y_{1}\right)\right]+\left[U\left(X \mid Y_{1}\right)-U(X \mid Y)\right] \\
& =\left[U(p)-\sum_{y} p_{\sigma}(y) U\left(p_{\sigma}(\cdot \mid y)\right)\right]+\left[\sum_{y} p_{\sigma}(y) U\left(p_{\sigma}(\cdot \mid y)\right)-p_{\sigma}\left(y, y^{j}\right) U\left(p_{\sigma}\left(\cdot \mid y y^{j}\right)\right)\right] \\
& =\left[U(p)-\sum_{y} p_{\sigma}(y) U\left(p_{\sigma}(\cdot \mid y)\right)\right]+\left[\sum_{y} p_{\sigma}(y) U\left(p_{\sigma}(\cdot \mid y)\right)-p_{\sigma}(y) p_{\sigma}\left(y^{j} \mid y\right) U\left(p_{\sigma}\left(\cdot \mid y y^{j}\right)\right)\right] \\
& =\left[U(p)-\sum_{y} p_{\sigma}(y) U\left(p_{\sigma}(\cdot \mid y)\right)\right]+\sum_{y} p_{\sigma}(y)\left[U\left(p_{\sigma}(\cdot \mid y)\right)-\sum_{y^{j}} p_{\sigma}\left(y^{j} \mid y\right) U\left(p_{\sigma}\left(\cdot \mid y y^{j}\right)\right)\right] \\
& =\left[U(p)-\sum_{y} p_{a}(y) U\left(p_{a}(\cdot \mid y)\right)\right]+\sum_{y} p_{a}(y)\left[U\left(p^{a y}\right)-\sum_{y^{j}} p_{\sigma_{y}}^{a y}\left(y^{j}\right) U\left(p_{\sigma_{y}}^{a y}\left(\cdot \mid y^{j}\right)\right)\right] \\
& =I_{a}(\mathcal{S} ; p)+\sum_{y} p_{a}(y) I_{\sigma_{y}}\left(\mathcal{S} ; p^{a y} \cdot\right)
\end{aligned}
$$

Let us say that a strategy $\sigma$ of length $l$ is optimal for $\mathcal{S}, p(\cdot)$ and $l$ if it maximizes $I_{\sigma}(\mathcal{S}, p)$ among all strategies of length $l$.

Corollary 6.2 (Bellman-type equation for optimal strategies). There is an optimal strategy $\sigma^{\star}$ of length $l$ for $\mathcal{S}$ and $p(\cdot)$ that satisfies the following equation

$$
I_{\sigma^{\star}}(\mathcal{S} ; p)=\max _{a}\left\{I_{a}(\mathcal{S} ; p)+\sum_{y: p_{a}(y)>0} p_{a}(y) I_{\sigma_{a, y}^{\star}}\left(\mathcal{S} ; p^{a y}\right)\right\}
$$

where $\sigma_{a, y}^{\star}$ is an optimal strategy of length $l-1$ for $\mathcal{S}$ and $p^{a y}(\cdot)$.

Corollary 6.2 allows us to employ dynamic programming or backward induction to compute optimal finite strategies. We discuss this briefly in the next subsection.

6.2. Markov Decision Processes and Backward Induction. A mechanism $\mathcal{S}$ and a prior $p(\cdot)$ induce a Markov Decision Process (MDP), where all possible attack trees are represented at once. Backward induction amounts to recursively computing the most efficient attack tree out of this MDP, limited to a given length. More precisely, the MDP $\mathcal{M}$ induced by $\mathcal{S}$ and a prior $p(\cdot)$ is an in general infinite tree consisting of decision nodes and probabilistic nodes. Levels of decision nodes alternate with levels of probabilistic nodes, starting from 


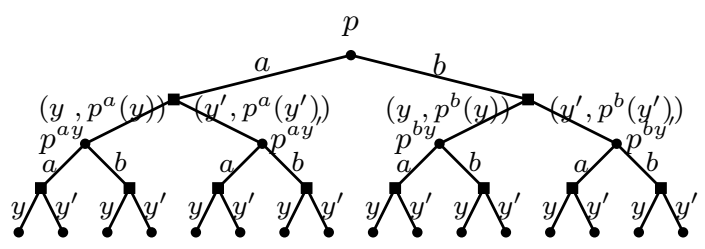

FiguRE 7 . The first few levels of a MDP induced by a prior $p(\cdot)$ and a mechanism with $A c t=\{a, b\}$ and $\mathcal{Y}=\left\{y, y^{\prime}\right\}$. Round nodes are decision nodes and squares nodes are probabilistic nodes. For the sake of space, labels of the last level of arcs and nodes are only partially shown.

the root which is a decision node. Decision nodes are labelled with probability distributions over $\mathcal{X}$, edges outgoing decision nodes with actions, and edges outgoing probabilistic nodes with pairs $(y, \lambda)$ of an observation and a real, in such a way that (again, we identify nodes with the corresponding history):

- a decision node corresponding to history $h$ is labelled with $p^{h}(\cdot)$, if this is defined, otherwise the node and its descendants are removed, as well as the incoming edge;

- for any pair of consecutive edges leading from a decision node $h$ to another decision node hay, for any $a \in A c t$ and $y \in \mathcal{Y}$, the edge outgoing the probabilistic node is labelled with $\left(y, p_{a}^{h}(y)\right)$.

Figure 7 shows the first few levels of such a MDP.

In order to compute an optimal strategy of length $l \geq 1$ by backward induction, one initially prunes the tree at $l$-th decision level (the root is at level 0 ) and then assigns rewards to all leaves of the resulting tree. Moreover, each probabilistic node is assigned an immediate gain. Rewards are then gradually propagated from the leaves up to the root, as follows:

- each probabilistic node is assigned as a reward the sum of its immediate gain and the average reward of its children, average computed using the probabilities on the outgoing arcs;

- each decision node is assigned the maximal reward of its children; the arc leading to the maximizing child is marked or otherwise recorded.

Eventually, the root will be assigned the maximal achievable reward. Moreover, the paths of marked arcs starting from the root will define an optimal strategy of length $l$. We can apply this strategy to our problem, starting with assigning rewards 0 to each leaf node $h$, and immediate gain $I_{a}\left(\mathcal{S}, p^{h}\right)$ to each $a$-child of any decision node $h$. The correctness of the resulting procedure is obvious in the light of Corollary 6.2.

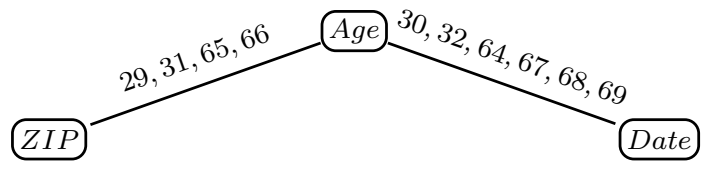

Figure 8. A Shannon entropy optimal strategy for Example 3.2. Leaves with the same label and their incoming arcs have been coalesced.

In a crude implementation of the above outlined procedure, the number of decision nodes in the MDP will be bounded by $(|\mathcal{Y}| \times|A c t|)^{l+1}-1$ (probabilistic nodes can be dispensed with, at the cost of moving incoming action labels to outgoing arcs.) Assuming that each distribution is stored in space $O(|\mathcal{X}|)$, the MDP can be built and stored in time and space 
$\mathcal{O}\left(|\mathcal{X}| \times(|\mathcal{Y}| \times|A c t|)^{l+1}\right)$. This is also the running time of the backward induction outlined above, assuming $U(\cdot)$ can be computed in time $O(|\mathcal{X}|)$ (some straightforward optimizations are possible here, but we will not dwell on this.) By comparison, the running time of the exhaustive procedure for deterministic systems outlined in [26, Th.1] is $O\left(l \times|A c t|^{r^{l}} \times|\mathcal{X}| \times\right.$ $\log |\mathcal{X}|$ ), where $r$ is the maximal number of classes in any relation $\equiv_{a}$; since $r$ can be as

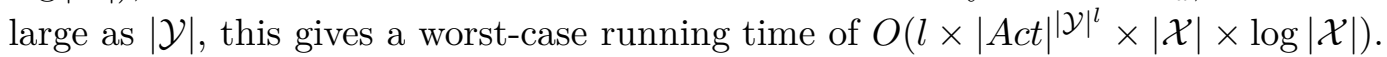

Example 6.3. Applying backward induction to the mechanism of Example 3.2 with $U(\cdot)=$ $H(\cdot)$ and $l=2$, one gets the optimal strategy $\sigma$ shown in Figure 8 , with $I_{\sigma}(\mathcal{S}, p) \approx 2.4$ bits. Some details of the derivation of this strategy are reported in Appendix $\mathbb{C}$.

\section{THE ROLE OF CONCAVITY}

We elucidate a connection between our definition of uncertainty (convexity + continuity) and a concept of scoring rule in Bayesian decision theory. A scoring rule encodes a system of (dis)incentives: a wrong forecast about an event causes the forecaster a loss, whose magnitude depends on both the forecast that has been put forward, and on the event that has actually occurred. The average loss under the best forecast is named entropy in this context. In essence, we will show that: (a) every proper scoring rule induces an entropy function that is concave, hence necessarily continuous at least in the interior of the probability simplex; (b) every concave function arises, under an additional mild assumption, as the entropy induced by a certain scoring rule. This almost complete correspondence, and its simple definition, give a strong support to our choice of the class of uncertainty functions.

The connection between concavity and uncertainty has been explored in Statistics at least starting from the 1950's, and it comes into many different flavours. The following discussion is our personal take of this issue, for which we claim no technical novelty. Our presentation is partly inspired by [17, Sections 9-10]. For notational simplicity, in what follows we fix any ordering of the elements of $\mathcal{X}$, say $x_{1}, \ldots, x_{n}$, so that we can identify any distribution $p$ with a vector $\left(p_{1}, \ldots, p_{n}\right) \in \mathbb{R}^{n}$.

Formally, in the context of Bayesian decision theory, a scoring rule is a function

$$
S: \mathcal{X} \times \stackrel{\circ}{\mathcal{P}} \rightarrow \mathbb{R}
$$

where $\stackrel{\circ}{\mathcal{P}}$ denotes here the interior part of $\mathcal{P}$, that is, the set of those distributions $q$ s.t. $q(x)>0$ for each $x \in \mathcal{X}$. This function is given the following interpretation. A forecaster (in our case, the adversary) is asked to put forward a forecast about the outcome of an event (in our case, the secret.) A forecast takes the form of a probability distribution $q$, which represents the forecaster's estimation of the probability of each possible outcome $x \in \mathcal{X}$. Then $S(x, q)$ represents the loss incurred by the forecaster when the outcome is actually $x$ and he has put forward $q$. If the outcome is distributed according to $p$, the average loss incurred if putting forward $q \in \stackrel{\circ}{\mathcal{P}}$ is given by

$$
S(p, q) \triangleq \sum_{x} p(x) S(x, q) .
$$

This definition is extended to each $q$ in the frontier, $q \in \mathcal{P} \backslash \stackrel{\circ}{\mathcal{P}}$, by $\liminf _{q^{\prime} \rightarrow q} S\left(p, q^{\prime}\right)$, be this finite or infinite. The scoring rule $S$ is called proper if the choice $q=p$ always minimizes $S(p, q)$. In other words, a proper scoring rule (PSR) encodes a penalty system that forces 
the forecaster to be honest and propose the distribution he really thinks is the true one. For a PSR, the minimal loss corresponding to $p$ is - perhaps not surprisingly - also called the induced entropy

$$
H(p) \triangleq S(p, p) .
$$

Seen as a function of $p, H(p)$ gives a measure of the intrinsic risk associated with each $p$, under the loss model encoded by the PSR $S$.

Proposition 7.1. Any entropy function induced by a PSR is a concave function over $\mathcal{P}$. As a consequence, it is continuous over $\stackrel{\circ}{\mathcal{P}}$.

Proof. Let $H(p)=S(p, p)$, with $S$ a PSR. Consider any $\lambda \in[0,1]$ and $p, q \in \mathcal{P}$, and let $r=\lambda p+(1-\lambda) q$. Assume first that $r$ is in the interior of $\mathcal{P}$. Then

$$
\begin{aligned}
H(r) & =S(r, r) \\
& =S(\lambda p+(1-\lambda) q, r) \\
& =\lambda S(p, r)+(1-\lambda) S(q, r) \\
& \geq \lambda S(p, p)+(1-\lambda) S(q, q) \\
& =\lambda H(p)+(1-\lambda) H(q)
\end{aligned}
$$

where the second equality follows from the linearity of $S$ w.r.t. the first argument and the inequality follows from the definition of PSR. If $r$ is in the frontier of $\mathcal{P}$, by the properties of lim inf, one has

$$
\begin{aligned}
H(r) & =\liminf _{r^{\prime} \rightarrow r} S\left(r, r^{\prime}\right) \\
& =\liminf _{r^{\prime} \rightarrow r} S\left(\lambda p+(1-\lambda), r^{\prime}\right) \\
& =\liminf _{r^{\prime} \rightarrow r} \lambda S\left(p, r^{\prime}\right)+(1-\lambda) S\left(q, r^{\prime}\right) \\
& \geq \lambda \liminf _{r^{\prime} \rightarrow r} S\left(p, r^{\prime}\right)+(1-\lambda) \liminf _{r^{\prime} \rightarrow r} S\left(q, r^{\prime}\right) \\
& =\lambda S(p, r)+(1-\lambda) S(q, r)
\end{aligned}
$$

and then the reasoning proceeds as above. This concludes the proof that $H$ is concave.

Finally, it is a standard result that concavity over $\mathcal{P}$ implies continuity - in fact, local lipschitzianity - over $\stackrel{\circ}{\mathcal{P}}$ (see e.g. [10].)

On the other hand, under a mild additional assumption, any concave function is induced by a PSR, as we will check shortly. Let $H$ be concave on $\mathcal{P}$. It is well known that concave functions enjoy the following supporting hyperplane property (see e.g. [10].) For each $q \in \stackrel{\circ}{\mathcal{P}}$, there exists a vector $c_{q}=\left(c_{1}, \ldots, c_{n}\right) \in \mathbb{R}^{n}$ such that, for each $p \in \mathcal{P}$ (here $\langle\cdot, \cdot\rangle$ denotes the usual scalar product between two vectors)

$$
H(p) \leq H(q)+\left\langle c_{q}, p-q\right\rangle=H(q)+\sum_{i=1}^{n} c_{i}\left(p_{i}-q_{i}\right) .
$$

The above relation merely means that the graph of $H$ is all below an hyperplane that is tangent to the point $(q, H(q))$. A vector $c_{q}$ satisfying (7.1) is called a subgradient of $H$ at $q$. In particular, if $H$ is differentiable at $q$, there is exactly one choice for $c_{q}$, namely the 
gradient of $H$ at $q$

$$
c_{q}=\nabla H(q)=\left(\frac{\partial H}{\partial x_{1}}(q), \ldots, \frac{\partial H}{\partial x_{n}}(q)\right) .
$$

Now, for $q \in \stackrel{\circ}{\mathcal{P}}$, we set

$$
S\left(x_{i}, q\right) \triangleq H(q)+c_{i}-\left\langle c_{q}, q\right\rangle .
$$

Clearly, for each $p$, we have $S(p, q)=E_{p}[S(X, q)]=H(q)+\left\langle c_{q}, p-q\right\rangle$. We extend this to $q$ in the frontier by $S(p, q) \triangleq \liminf _{q^{\prime} \rightarrow q} S\left(p, q^{\prime}\right)$. Assume now that the vectors $c_{q}$ can be chosen in such a way that the following property holds true for any $p$ in the frontier

$$
\liminf _{p^{\prime} \rightarrow p}\left\langle c_{p^{\prime}}, p-p^{\prime}\right\rangle=0 \text {. }
$$

This condition means that, as $p^{\prime}$ approaches a point $p$ in the frontier, the tangent hyperplane at $p^{\prime}$ does not approach a vertical hyperplane "too fast". We now check that $S(x, q)$ is a PSR. First, note that for any $p \in \mathcal{P}$, we have $S(p, p)=H(p)$. For $p$ in the interior, this follows by definition; while for $p$ in the frontier, we note that $S(p, p)=\liminf _{p^{\prime}} S\left(p, p^{\prime}\right)=$ $H(p)+\lim \inf _{q^{\prime}}\left\langle c_{p^{\prime}}, p-p^{\prime}\right\rangle$ and then exploit (17.3). Now, applying the supporting hyperplane property (7.1) for $q$ in the interior we have

$$
\begin{aligned}
S(p, q) & =E_{p}[S(X, q)] \\
& =H(q)+\left\langle c_{q}, p-q\right\rangle \\
& \geq H(p) \\
& =S(p, p) .
\end{aligned}
$$

For $q$ in the frontier, the same property follows from

$$
S(p, q)=\liminf _{q^{\prime}} S\left(p, q^{\prime}\right) \geq \liminf _{q^{\prime}} S(p, p)=S(p, p),
$$

where the inequality follows from above. This shows that $S(x, q)$ is a PSR and that the induced entropy is precisely the given concave function $H$. In other words, we have just shown the following proposition.

Proposition 7.2. Every concave function over $\mathcal{P}$ that respects (7.3) is the entropy induced by some PSR.

Example 7.3. Let us consider the Shannon entropy function $H(p)=-\sum_{i} p_{i} \log p_{i}$. By direct calculation, it is immediate to check that $S\left(x_{i}, q\right)=-\log q_{i}$ is a PSR for Shannon entropy. However, it is instructive to apply the recipe in the proof of Proposition 7.2 to reconstruct $S(x, q)$.

$H(p)$ is differentiable in the interior of the probability simplex. The gradient of $H$ at $q$ is

$$
\nabla H(q)=\left(-\left(1+\log q_{1}\right), \ldots,-\left(1+\log q_{n}\right)\right) \triangleq c_{q} .
$$

Now easy calculations show that $\left\langle c_{p^{\prime}}, p-p^{\prime}\right\rangle=H(p)-H\left(p^{\prime}\right)+D\left(p \| p^{\prime}\right) \rightarrow 0$ as $p^{\prime} \rightarrow p$, thus making the condition (7.3) true7. Noting that $\left\langle c_{q}, q\right\rangle=H(q)-1$, we apply (17.2) and define

$$
S\left(x_{i}, q\right) \triangleq H(q)-\left(1+\log q_{i}\right)-(H(q)-1)=-\log q_{i} .
$$

\footnotetext{
${ }^{7}$ Here $D\left(p \| p^{\prime}\right) \triangleq \sum_{i} p_{i} \log \left(p_{i} / q_{i}\right)$ is the familiar Kullback-Leibler divergence. Note in particular that $D\left(p \| p^{\prime}\right) \rightarrow 0$ as $p^{\prime} \rightarrow p$. In passing, it is not true in general that $D\left(p \| p^{\prime}\right) \rightarrow 0$ as $p \rightarrow p^{\prime}$.
} 
A similar calculation for $H(p)=\operatorname{var}(p)$ yields

$$
S\left(x_{i}, q\right)=\operatorname{var}(q)+x_{i}^{2}-2 x_{i}-E_{q}\left[X^{2}\right]+2 E_{q}[X]=\left(x_{i}-1\right)^{2}-\left(E_{q}[X]-1\right)^{2}
$$

which leads to $S(p, q)=-E_{q}[X]^{2}+2 E_{q}[X]+E_{p}\left[X^{2}\right]-2 E_{p}[X]$ and, as expected, $S(p, p)=$ $\operatorname{var}(p)$.

We finally consider the case of error entropy, $H(p)=1-\max _{i} p_{i}$. For each $q$ let us denote by $j_{q}$ an index in $\{1, \ldots, n\}$ such that $q_{j_{q}}=\max _{i} q_{i}$ (if there is more than one such index, choose one arbitrarily.) At each $q$ with a single maximal element, $H$ is differentiable and we can set (here $\delta_{x y}$ is the Kronecker's delta symbol)

$$
c_{q} \triangleq \nabla H(q)=\left(-\delta_{1 j_{q}}, \ldots,-\delta_{n j_{q}}\right) .
$$

Hence, applying (7.2) and noting that $\left\langle c_{q}, q\right\rangle=-q_{j_{q}}$, for each such $q$ we let

$$
S\left(x_{i}, q\right) \triangleq\left(1-q_{j_{q}}\right)-\delta_{i j_{q}}+q_{j_{q}}=1-\delta_{i j_{q}} .
$$

At points $q$ with more than one maximal element, $H$ is not differentiable, and the choice of $c_{q}$ is not unique. However, $c_{q}=\left(-\delta_{1 j_{q}}, \ldots,-\delta_{n j_{q}}\right)$ is still a subgradient, so the same definition of $S\left(x_{i}, q\right)$ as above applies. Note that $S(p, q)=1-p_{j_{q}}$ and, in particular, $S(p, p)=1-p_{j_{p}}=1-\max _{i} p_{i}$ as expected.

\section{Conclusion, Related and Further Work}

We have proposed a general information-theoretic model for the analysis of confidentiality under adaptive attackers. Within this model, we have proven several results on the limits of such attackers, on the relations between adaptive and non-adaptive strategies, and on the problem of searching for optimal finite strategies. We have also elucidated a connection between our notion of uncertainty function and Bayesian decision theory.

8.1. Related Work. In [26], Köpf and Basin introduced an information-theoretic model of adaptive attackers for deterministic mechanisms. Their analysis is conducted essentially on the case of uniform prior distributions. Our model generalizes [26] in several respects: we consider probabilistic mechanisms, generic priors and generic uncertainty functions. More important than that, we contrast quantitatively the efficiency of adaptive and non-adaptive strategies, we characterize maximum leakage of infinite strategies, and we show how to express information leakage as a Bellman equation. The latter leads to search algorithms for optimal strategies that, when specialized to the deterministic case, are more time-efficient than the exhaustive search outlined in [26] (see Section 6.)

Our previous paper [7] tackles multirun, non-adaptive adversaries, in the case of minentropy leakage. In this simpler setting, a special case of the present framework, one manages to obtain simple analytical results, such as the exact convergence rate of the adversary's success probability as the number of observations goes to infinity.

Alvim et al. [1] study information flow in interactive mechanisms, described as probabilistic automata where secrets and observables are seen as actions that alternate during execution. Information-theoretically, they characterize these mechanisms as channels with feedback, giving a Shannon-entropy based definition of leakage. Secret actions at each step depend on previous history, but it is not clear that this gives the adversary any ability to adaptively influence the next observation, in our sense. 
In [2], Alvim et al. study g-leakage, a generalization of min-entropy leakage, where the adversary's benefit deriving from a guess about a secret is specified using a gain function $g$ : intuitively, the closer the guess to the secret, the higher the gain. Alvim et al. derive general results about $g$-leakage, including bounds between min-capacity, $g$-capacity and Shannon capacity. Gain functions are conceptually very close to the proper scoring rules (PSRs) we considered in Section 7. Abstracting from the unimportant difference of encoding gains rather then losses, a gain function can be seen in fact as a special case of a PSR where the forecast put forward by the forecaster is always a Dirac's delta. One important technical difference between [2] on one side and the framework of PSRs and our paper on the other side, is that entropy functions, as defined in [2], are all generalizations of the familiar min-entropy. As such, they are in general neither concave nor convex. A thorough investigation of the connections between $g$-leakage and PRSs is left for future work. More or less contemporary to the short version of the present paper is Mardziel et al.'s [29], which extends the analysis via $g$-leakage functions to systems with memory. This work is similar in spirit to ours, but now successive responses to queries may not be independent, as the secret evolves over time. They too utilize backward induction to calculate leakage. Like in the static case, this dynamic $g$-leakage does not lend itself to be recast in the present framework. A dynamic approach is also at the core of a model in [5] based on Hidden Markov Models, where the observed system evolves over time, although the secret is fixed.

After the publication of the short version of the present paper [8], we learned from a statistician colleague about the existence of a large body of work in Bayesian forecasting and PSRs. A good synthesis of this research can be found in the works of DeGroot, see e.g. [18, 19] and references therein, although the terminology used there is slightly different (utility functions are considered rather than scoring rules.) Remarkably, [18] contains considerations on sequential observations and decisions which, despite the different terminology and emphases, come very close to our adaptive model of QIF. Dawid [17] and Gneiting and Raftery [24] give modern accounts of these themes. The role of concavity in QIF is also central to some recent works by McIver et al. [30, 31]. An important result is the presentation of a prior vulnerability as a "disorder test" that is, interestingly, defined in terms of continuous and concave functions. It would be interesting to see how much these approaches share with the one based on PSRs.

The use of Bellman equation and backward induction, applied to multi-threaded programs, in a context where strategies are schedulers, is also found in [13]. [28, 23] propose models to assess system security against classes of adversaries characterized by user-specified 'profiles'. While these models share some similarities with ours - in particular, they too employ MDP's to keep track of possible adversary strategies - their intent is quite different from ours: they are used to build and assess analysis tools, rather than to obtain analytical results. Also, the strategies they consider are tailored to worst-case adversary's utility, which, differently from our average-case measures, is not apt to express information leakage.

8.2. Further work. There are several directions worth being pursued, starting from the present work. First, one would like to implement and experiment with the search algorithm described in Section 6. Adaptive querying of datasets might represent an ideal ground for evaluation of such algorithms. Second, one would like to investigate worst-case variations of the present framework: an interesting possibility is to devise an adaptive version of Differential Privacy [21, 22] or one of its variants [9]. Finally, connections between (adaptive) QIF and PSRs in Statistics deserve to be further explored. 


\section{ACKNOWLEDGEMENT}

We should like to thank Fabio Corradi at DiSIA for stimulating discussions on the relationship between information leakage and inference in Statistics, and in particular for pointing out to us the work on scoring rules in Bayesian decision theory.

We thank an anonymous reviewer of [8] for suggesting us the envelopes example (Example 4.4).

\section{REFERENCES}

[1] M.S. Alvim, M.E. Andrés, C. Palamidessi. Information Flow in Interactive Systems. Journal of Computer Security, 20(1): 3-50, 2012.

[2] M.S. Alvim, K. Chatzikokolakis, C. Palamidessi, G. Smith. Measuring Information Leakage Using Generalized Gain Functions. IEEE 25th Computer Security Foundations Symposium 2012, IEEE Computer Society: 265-279, 2012.

[3] M. Backes, B. Köpf. Formally Bounding the Side-Channel Leakage in Unknown-Message Attacks. ESORICS 2008, LNCS 5283: 517-532, Springer, 2008.

[4] M. Boreale. Quantifying information leakage in process calculi. Information and Computation 207(6): 699-725, 2009.

[5] M. Boreale, F. Pampaloni, M. Paolini. Asymptotic information leakage under one-try attacks. Proc. of FoSSaCS 2011, LNCS 6604: 396-410, Springer, 2011. Full version in Mathematical Structures in Computer Science 25(2): 292-319, 2015.

[6] M. Boreale, F. Pampaloni, M. Paolini. Quantitative information flow, with a view. Proc. of ESORICS 2011, LNCS 6879: 588-606, Springer, 2011.

[7] M. Boreale, F. Pampaloni. Quantitative multirun security under active adversaries. Proc. of QEST 2012: 158-167, IEEE, 2012.

[8] M. Boreale, F. Pampaloni. Quantitative Information Flow under Generic Leakage Functions and Adaptive Adversaries.Proc. of FORTE 2014, LNCS 8461:166-181, Springer, 2014.

[9] M. Boreale, M. Paolini. Worst- and Average-Case Privacy Breaches in Randomization Mechanisms. IFIP TCS 2012, LNCS 7604: 72-86, Springer, 2012.

[10] S. Boyd, L. Vandenberghe. Convex Optimization. Cambridge University Press, 2004.

[11] C. Braun, K. Chatzikokolakis, C. Palamidessi. Quantitative Notions of Leakage for One-try Attacks. Proc. of MFPS 2009, Electr. Notes Theor. Comput. Sci. 249: 75-91, 2009.

[12] K. Chatzikokolakis, C. Palamidessi, P. Panangaden. Anonimity Protocols as Noisy Channels. Information and Computation, 206(2-4): 378-401, 2008.

[13] H. Chen, P. Malacaria. The Optimum Leakage Principle for Analyzing Multi-threaded Programs. ICITS 2009, LNCS 5973:177-193, Springer, 2009.

[14] D. Clark, S. Hunt, P. Malacaria. Quantitative Analysis of the Leakage of Confidential Data. Electr. Notes Theor. Comput. Sci. 59(3), 2001.

[15] M. R. Clarkson, F. B. Schneider. Quantification of Integrity. Mathematical Structures in Computer Science 25(2): 207-258, 2015.

[16] T. M. Cover, J. A. Thomas. Elements of Information Theory, 2/e. John Wiley \& Sons, 2006.

[17] A. P. Dawid. Coherent measures of discrepancy, uncertainty and dependence, with applications to bayesian predictive experimental design. Department of Statistical Science, University College London. http://www.ucl.ac.uk/Stats/research/abs94.html, Tech. Rep. 139, 1998.

[18] M. H. DeGroot. Uncertainty, Information, and Sequential Experiments. Ann. Math. Statist. 33(2), p. 404-419, 1962.

[19] M. H. DeGroot. Optimal Statistical Decisions, WCL edition. John Wiley \& Sons, 2004.

[20] Y. Dodis, A. Smith. Entropic Security and the Encryption of High Entropy Messages. TCC 2005, LNCS 3378, Springer, Heidelberg, 2005.

[21] C. Dwork. Differential Privacy. ICALP 2006 LNCS 4052: 1-12, 2006.

[22] C. Dwork, F. McSherry, K. Nissim, A. Smith. Calibrating Noise to Sensitivity in Private Data Analysis. Proc. of the 3rd IACR Theory of Cryptography Conference, 2006. 
[23] M.D. Ford, P. Buchholz, W.H. Sanders. State-Based Analysis in ADVISE. Proc. of QEST 2012, pp. 148-157, IEEE, 2012.

[24] T. Gneiting, A.E. Raftery. Strictly proper scoring rules, prediction, and estimation. Journal of the American Statistical Association, 102: 359-378, 2007.

[25] J. Kelsey, B. Schneier, D. Wagner, C. Hall. Side Channel Cryptanalysis of Product Ciphers. Journal of Computer Security 8(2/3): 2000.

[26] B. Köpf, D. Basin. An Information-Theoretic Model for Adaptive Side-Channel Attacks. ACM Conference on Computer and Communications Security 2007:286-296, 2007.

[27] B. Köpf, G. Smith. Vulnerability Bounds and Leakage Resilience of Blinded Cryptography under Timing Attacks. CSF 2010: 44-56, 2010.

[28] E. LeMay, M.D. Ford, K. Keefe, W.H. Sanders, C. Muehrcke. Model-based Security Metrics using ADversary VIew Security Evaluation (ADVISE.) Proc. of QEST 2011, pp. 191-200, IEEE, 2011.

[29] P. Mardziel, M. S. Alvim, M. W. Hicks, M. R. Clarkson. Quantifying Information Flow for Dynamic Secrets. IEEE Symposium on Security and Privacy 2014: 540-555, IEEE, 2014.

[30] A. McIver, L. Meinicke, C. Morgan. A Kantorovich-Monadic Powerdomain for Information Hiding, with Probability and Nondeterminism. LICS 2012: 461-470, IEEE, 2012.

[31] A. McIver, C. Morgan, G. Smith, B. Espinoza, L. Meinicke. Abstract Channels and Their Robust Information-Leakage Ordering. POST 2014, LNCS 8414: 83-102, Springer, 2014.

[32] G. Smith. On the Foundations of Quantitative Information Flow. Proc. of FoSSaCS 2009, LNCS 5504: 288-302, 2009.

\section{Appendix A. Proofs of Lemma 2.7 and of Lemma 5.2}

Lemma A.1 (Lemma 2.7). $I_{\sigma}(X ; Y) \geq 0$. Moreover $I_{\sigma}(X ; Y)=0$ if $X$ and $Y$ are independent.

Proof. First note that, by a simple manipulation relying on Bayes theorem, we can express the prior distribution $p(\cdot)$ as follows, for each $x \in \mathcal{X}$ (summation ranges over $y$ of positive probability):

$$
p(x)=\sum_{y} p(y) p(x \mid y) .
$$

Hence by concavity of $U$ and Jensens's inequality:

$$
\begin{aligned}
U(X) & =U(p) \\
& =U\left(\sum_{y} p(y) p(\cdot \mid y)\right) \\
& \geq \sum_{y} p(y) U(p(\cdot \mid y)) \\
& =U(X \mid Y) .
\end{aligned}
$$

Moreover, if $X$ and $Y$ are independent, then for each $y$ of positive probability, $p(x)=p(x \mid y)$, hence $\sum_{y} p(y) p(\cdot \mid y)=p(\cdot)$, so that $U(X)=U(p)=U(X \mid Y)$.

Lemma A.2 (Lemma 5.2). $x \equiv x^{\prime}$ iff for each $a \in$ Act, $p_{a}(\cdot \mid x)=p_{a}\left(\cdot \mid x^{\prime}\right)$. In other words, $\equiv$ is $\cap_{a \in A c t} \equiv_{a}$.

Proof. Let $x, x^{\prime} \in \mathcal{X}$. Assume first $x \equiv_{a} x^{\prime}$ for each action $a$. Let $\sigma$ be any finite strategy and $y^{n} y_{n+1}$ a sequence such that $y^{n} \in \operatorname{dom}(\sigma)$ and $y^{n} y_{n+1} \notin \operatorname{dom}(\sigma)$. From (2.1), we know that

$$
\begin{aligned}
p_{\sigma}\left(y^{n} y_{n+1} \mid x\right) & =p_{a_{1}}\left(y_{1} \mid x\right) \cdots p_{a_{n}}\left(y_{n} \mid x\right) p_{a_{n+1}}\left(y_{n+1} \mid x\right) \\
p_{\sigma}\left(y^{n} y_{n+1} \mid x^{\prime}\right) & =p_{a_{1}}\left(y_{1} \mid x^{\prime}\right) \cdots p_{a_{n}}\left(y_{n} \mid x^{\prime}\right) p_{a_{n+1}}\left(y_{n+1} \mid x^{\prime}\right)
\end{aligned}
$$


where $a_{1}=\sigma(\epsilon), a_{2}=\sigma\left(y_{1}\right), \ldots, a_{n}=\sigma\left(y_{1} \cdots y_{n-1}\right)$ and $a_{n+1}=\sigma\left(y^{n}\right)$. From the above equations, it is immediate to conclude that, as $p_{a}(\cdot \mid x)=p_{a}\left(\cdot \mid x^{\prime}\right)$ for each action $a$, then $p_{\sigma}\left(y^{n} y_{n+1} \mid x\right)=p_{\sigma}\left(y^{n} y_{n+1} \mid x^{\prime}\right)$. Since this holds for any finite $\sigma$, we conclude $x \equiv x^{\prime}$. The other direction is obvious, as $p_{a}(\cdot \mid x)=p_{[a]}(\cdot \mid x)$ (where $[a]$ is a length 1 strategy) for any $a$ and $x$.

\section{Appendix B. Proof of Theorem 5.5}

In order to prove Theorem [5.5, we introduce some terminology and concepts from the information-theoretic method of types [16]. For distributions $p(\cdot)$ and $q(\cdot)$ on $\mathcal{Y}$, we let their Kullback-Leibler $(K L)$ divergence be defined as: $D(p \| q) \triangleq \sum_{y} p(y) \log \frac{p(y)}{q(y)}$, with the proviso that $0 \cdot \log \frac{0}{p(y)}=0$ and $p(y) \cdot \log \frac{p(y)}{0}=+\infty$ for $p(y)>0$. Given $n \geq 1$, and a sequence $y^{n} \in$ $\mathcal{Y}^{n}$, the type (or empirical distribution) of $y^{n}$, denoted $t_{y^{n}}$, is the probability distribution over $\mathcal{Y}$ defined thus: $t_{y^{n}}(y) \triangleq \frac{\mathrm{n}\left(y \mid y^{n}\right)}{n}$, where $\mathrm{n}\left(y \mid y^{n}\right)$ denotes the number of occurrences of $y$ in $y^{n}$. In this section, $H$ will always stand for Shannon entropy, $H(p)=-\sum_{x} p(x) \log p(x)$. We will often abbreviate $H\left(t_{y^{n}}\right)$ as $H\left(y^{n}\right)$, and $D\left(t_{y^{n}} \| q\right)$ as $D\left(y^{n} \| q\right)$, thus denoting the type by a corresponding sequence, when no confusion arises. Given $\varepsilon>0$ and a probability distribution $q(\cdot)$ on $\mathcal{Y}$, the "ball" of $n$-sequences whose type is within distance $\varepsilon$ of $q(\cdot)$ is defined thus:

$$
B^{(n)}(q, \varepsilon) \triangleq\left\{y^{n}: D\left(y^{n} \| q\right) \leq \varepsilon\right\} .
$$

We shall also make use of the following new terminology about sequences. Assume $|A c t|=$ $k$. Given a sequence $y^{n}=\left(y_{1}, y_{2}, \ldots, y_{n}\right)$ and an integer $j=1, \ldots, k$, we shall denote by $y^{n}(j)$ the subsequence $\left(y_{j}, y_{k+j}, y_{2 k+j}, \ldots\right)$, obtained by taking the symbols of $y^{n}$ at position $j, k+j, 2 k+j, \ldots$. In the rest of the section, unless otherwise stated, we let $\sigma$ be the infinite non-adaptive strategy that plays actions $a_{1}, \ldots, a_{k}, a_{1}, a_{2}, \ldots$, in a lock-step fashion: $\sigma\left(y^{j}\right) \triangleq a_{(j \bmod k)+1}$. For any $n \geq 1$, we let $\sigma_{n}$ be the truncation at level $n$ of $\sigma$ : $\sigma_{n} \triangleq \sigma \backslash n$. For a prior $p(\cdot)$, let $p_{\sigma_{n}}$ be the resulting joint probability distribution on $\mathcal{X} \times \mathcal{Y}^{n}$ : note that, for each $x$, the support of $p_{\sigma_{n}}(\cdot \mid x)$ is included in $\mathcal{Y}^{n}$. Let $\left(X, Y^{n}\right)$ be jointly distributed according to $p_{\sigma_{n}}$ : here we have introduced the superscript ${ }^{n}$ to record explicitly the dependence of $Y$ from $n$. Let us define the set of sequences $y^{n}$ where the type of each sub-sequence $y^{n}(i)$ is within $\varepsilon$ distance of the distribution $p_{a_{i}}(\cdot \mid x)$, thus:

$$
\hat{B}^{(n)}(x, \varepsilon) \triangleq\left\{y^{n}: D\left(y^{n}(i)|| p_{a_{i}}(\cdot \mid x)\right) \leq \varepsilon \text { for } i=1, \ldots, k\right\} .
$$

Furthermore, we define the following quantities depending on a given sequence $y^{n}$ and $x \in \mathcal{X}$ :

$$
\begin{aligned}
\hat{H}\left(y^{n}\right) & \triangleq \sum_{i=1}^{k} H\left(y^{n}(i)\right) \\
\hat{D}\left(y^{n}|| p_{\sigma_{n}}(\cdot \mid x)\right) & \triangleq \sum_{i=1}^{k} D\left(y^{n}(i) \| p_{a_{i}}(\cdot \mid x)\right) .
\end{aligned}
$$


Finally, for each sequence $y^{m} \in \mathcal{Y}^{m}$ and for each action $a \in A c t$, we let

$$
p_{a}^{m}\left(y^{m} \mid x\right)=\prod_{i=1}^{m} p_{a}\left(y_{i} \mid x\right)
$$

(this is the probability of generating $y^{m}$ with i.i.d. extractions obeying distribution $p_{a}(\cdot \mid x)$.)

Lemma B.1. Let $n$ be a multiple of $k$ and $x \in \mathcal{X}$. Then

$$
p_{\sigma_{n}}\left(y^{n} \mid x\right)=2^{-\frac{n}{k}\left[\hat{H}\left(y^{n}\right)+\hat{D}\left(y^{n} \| p_{\sigma_{n}}(\cdot \mid x)\right)\right]} .
$$

Proof.

$$
\begin{aligned}
p_{\sigma_{n}}\left(y^{n} \mid x\right) & =\prod_{j=1}^{k} p_{a_{j}}^{\frac{n}{k}}\left(y^{n}(j) \mid x\right) \\
& =\prod_{j=1}^{k} 2^{-\frac{n}{k}\left(H\left(y^{n}(j)\right)+D\left(y^{n}(j) \| p_{a_{j}}(\cdot \mid x)\right)\right)} \\
& =2^{-\frac{n}{k} \sum_{j=1}^{k}\left(H\left(y^{n}(j)\right)+D\left(y^{n}(j) \| p_{a_{j}}(\cdot \mid x)\right)\right)} \\
& =2^{-\frac{n}{k}\left(\hat{H}\left(y^{n}\right)+\hat{D}\left(y^{n} \| p_{\sigma_{n}}(\cdot \mid x)\right)\right)}
\end{aligned}
$$

where: (B.5) follows from re-arranging factors and the definition of $p_{a_{j}}^{\frac{n}{k}}(\cdot)$; (B.6) follows from [16, Theorem 11.1.2]; in (B.7) we have applied definitions (B.2) and (B.3).

Below, for a set $A$ and a distribution $q(\cdot)$, we let $q(A)$ denote $\sum_{a \in A} q(a)$.

Lemma B.2. Let $n$ be a multiple of $k, x \in \mathcal{X}$ and $\varepsilon>0$. Then

$$
p_{\sigma_{n}}\left(\hat{B}^{(n)}(x, \varepsilon) \mid x\right) \geq 1-2^{-\frac{n}{k} \varepsilon}\left(\frac{n}{k}+1\right)^{k|\mathcal{Y}|} C
$$

for some constant $C$, not depending on $n$.

Proof. Let $m=n / k$. We give a lower bound on the probability of $\hat{B}^{(n)}(x, \varepsilon)$ as follows.

$$
\begin{aligned}
p_{\sigma_{n}}\left(\hat{B}^{(n)}(x, \varepsilon) \mid x\right) & =\sum_{y^{n} \in \hat{B}^{(n)}(x, \varepsilon)} p_{\sigma_{n}}\left(y^{n} \mid x\right)=\sum_{y^{n} \in \hat{B}^{(n)}(x, \varepsilon)} \prod_{i=1}^{k} p_{a_{i}}^{m}\left(y^{n}(i) \mid x\right) \\
& =\prod_{i=1}^{k} \sum_{y^{m} \in B^{(m)}\left(p_{a_{i}}^{m}(\cdot \mid x), \varepsilon\right)} p_{a_{i}}\left(y^{m} \mid x\right)=\prod_{i=1}^{k} p_{a_{i}}^{m}\left(B^{(m)}\left(p_{a_{i}}(\cdot \mid x), \varepsilon\right) \mid x(\mathbf{B} .8)\right. \\
& \geq \prod_{i=1}^{k}\left(1-2^{-m \varepsilon}(m+1)^{|\mathcal{Y}|}\right)=\left(1-2^{-m \varepsilon}(m+1)^{|\mathcal{Y}|}\right)^{k} \\
& \left.=1+\sum_{i=1}^{k}\left(\begin{array}{c}
k \\
i
\end{array}\right)(-1)^{i} 2^{-m i \varepsilon}(m+1)^{|\mathcal{Y}| i} \geq 1-2^{-m \varepsilon}(m+1)^{k|\mathcal{Y}|} \text { (B. } 10\right)
\end{aligned}
$$

where: the first equality in (B.8) follows from the definition of $\hat{B}^{(n)}(x, \varepsilon)$; the inequality (B.9) follows from [16, Eq. 11.67]; in (B.10), $C=k \cdot \max _{i}\left(\begin{array}{c}k \\ i\end{array}\right)$ (note that $\left(\begin{array}{c}k \\ i\end{array}\right)$ is maximum when $i=\lceil k / 2\rceil$.) 
Lemma B.3. Let $x, x^{\prime} \in \mathcal{X}$, with $x \not \equiv x^{\prime}$. Let $n \geq 1$. Then there is $\varepsilon>0$ such that $\hat{B}^{(n)}(x, 2 \varepsilon) \cap \hat{B}^{(n)}\left(x^{\prime}, 2 \varepsilon\right)=\emptyset$.

Proof. It is well-known that given any two distinct distributions $p(\cdot)$ and $q(\cdot)$, there is $\varepsilon>0$ such that $B^{(n)}(p, 2 \varepsilon) \cap B^{(n)}(q, 2 \varepsilon)=\emptyset$ (this is a consequence of Pinsker's inequality, [16, Lemma 11.6.1].) Thus, choose $\varepsilon>0$ such that, for some $j, B^{(n)}\left(p_{a_{j}}(\cdot \mid x), 2 \varepsilon\right) \cap$ $B^{(n)}\left(p_{a_{j}}\left(\cdot \mid x^{\prime}\right), 2 \varepsilon\right)=\emptyset$ : the wanted statement follows from the definition of $\hat{B}^{(n)}(x, 2 \varepsilon)$ and $\hat{B}^{(n)}\left(x^{\prime}, 2 \varepsilon\right)$.

We are now set to prove Theorem 5.5 .

Theorem B.4 (Theorem 5.5). There is a total, non-adaptive strategy $\sigma$ s.t. $I_{\sigma}(\mathcal{S}, p)=$ $I(X ;[X])$. Consequently, $I_{\star}(\mathcal{S}, p)=I(X ;[X])$.

Proof. Using the notation previously introduced, we shall prove that, as $n \rightarrow \infty$

$$
U\left(X \mid Y^{n}\right) \longrightarrow L \quad \text { for some } L \leq U(X \mid[X]) .
$$

This will imply the thesis, as then $I_{\sigma}(\mathcal{S}, p) \geq I(X ;[X])$, which, by virtue of Theorem $[5.3$, implies $I_{\sigma}(\mathcal{S}, p)=I(X ;[X])$.

Let the equivalence classes of $\equiv$ be $c_{1}, \ldots, c_{K}$. For each $i=1, \ldots, K$, choose a representative $x_{i} \in c_{i}$ of nonzero probability (if it exists; otherwise class $c_{i}$ is just discarded.) We can compute as follows.

$$
\begin{aligned}
U\left(X \mid Y^{n}\right) & =\sum_{y^{n}, x} p(x) p_{\sigma_{n}}\left(y^{n} \mid x\right) U\left(p_{\sigma_{n}}\left(\cdot \mid y^{n}\right)\right) \\
& =\sum_{x} p(x) \sum_{y^{n}} p_{\sigma_{n}}\left(y^{n} \mid x\right) U\left(p_{\sigma_{n}}\left(\cdot \mid y^{n}\right)\right) \\
& \leq \sum_{x} p(x) U\left(\sum_{y^{n}} p_{\sigma_{n}}\left(y^{n} \mid x\right) p_{\sigma_{n}}\left(\cdot \mid y^{n}\right)\right) \\
& =\sum_{c_{i}} \sum_{x \in c_{i}} p(x) U\left(\sum_{y^{n}} p_{\sigma_{n}}\left(y^{n} \mid x_{i}\right) p_{\sigma_{n}}\left(\cdot \mid y^{n}\right)\right) \\
& =\sum_{c_{i}} p\left(c_{i}\right) U \underbrace{\left(\sum_{y^{n}} p_{\sigma_{n}}\left(y^{n} \mid x_{i}\right) p_{\sigma_{n}}\left(\cdot \mid y^{n}\right)\right)}_{q_{i}^{n}(\cdot)} \\
& =\sum_{c_{i}} p\left(c_{i}\right) U\left(q_{i}^{n}\right)
\end{aligned}
$$

where the inequality in (B.12) stems from $U$ 's concavity and Jensen's inequality. We will now show that there is a sub-sequence of indices $\left\{n_{j}\right\}$ such that for each $i=1, \ldots, K$,

$$
q_{i}^{n_{j}}(\cdot) \longrightarrow p\left(\cdot \mid c_{i}\right)
$$

(according to any chosen metrics in $\mathcal{P}$.) This will imply $(\mathrm{B} .11)$ : in fact, by virtue of $U$ 's continuity, we will have, on the chosen sub-sequence, $\sum_{c_{i}} p\left(c_{i}\right) U\left(q_{i}^{n_{j}}\right) \rightarrow \sum_{c_{i}} p\left(c_{i}\right) U\left(p\left(\cdot \mid c_{i}\right)\right)=$ $U(X \mid[X])$. Hence, by virtue of (B.13), on the chosen sub-sequence and hence on every sequence, we will have $U\left(X \mid Y^{n}\right) \rightarrow L \leq U(X \mid[X])$, which is (B.11). 
In order to prove ( $(\underline{\mathrm{B} .14})$, take any $n \geq 1$ that is a multiple of $k$, and choose any $\varepsilon>0$ such that $\hat{B}^{(n)}(x, 2 \varepsilon) \cap \hat{B}^{(n)}\left(x^{\prime}, 2 \varepsilon\right)=\emptyset$ whenever $x \not \equiv x^{\prime}$ (the existence of such an $\varepsilon$ is guaranteed by Lemma B.3.) Consider a generic $x \in c_{i}$ such that $p(x)>0$. We have the following lower bound for $q_{i}^{n}(x)$.

$$
\begin{aligned}
q_{i}^{n}(x) & =\sum_{y^{n}} \frac{p_{\sigma_{n}}\left(y^{n} \mid x_{i}\right) p_{\sigma_{n}}\left(y^{n} \mid x_{i}\right) p(x)}{\sum_{x^{\prime}} p_{\sigma_{n}}\left(y^{n} \mid x^{\prime}\right) p\left(x^{\prime}\right)}=\sum_{y^{n}} \frac{p_{\sigma_{n}}\left(y^{n} \mid x_{i}\right)}{\frac{p\left(c_{i}\right)}{p(x)}+\sum_{x^{\prime} \not x_{i}} \frac{p_{\sigma_{n}}\left(y^{n} \mid x^{\prime}\right) p\left(x^{\prime}\right)}{p_{\sigma_{n}}\left(y^{n} \mid x_{i}\right) p\left(x_{i}\right)}} \\
& \geq \sum_{y^{n} \in \hat{B}^{(n)}(x, \varepsilon)} \frac{p_{\sigma_{n}}\left(y^{n} \mid x_{i}\right)}{\frac{p\left(c_{i}\right)}{p(x)}+\sum_{x^{\prime} \neq x_{i}} 2^{-\frac{n}{k}\left[\hat{D}\left(y^{n}|| p_{\sigma_{n}}\left(\cdot \mid x^{\prime}\right)\right)-\hat{D}\left(y^{n}|| p_{\sigma_{n}}(\cdot \mid x)\right)\right] \frac{p\left(x^{\prime}\right)}{p(x)}}} \\
& \geq \sum_{y^{n} \in \hat{B}^{(n)}(x, \varepsilon)} \frac{p_{\sigma_{n}}\left(y^{n} \mid x_{i}\right)}{\frac{p\left(c_{i}\right)}{p(x)}+\sum_{x^{\prime} \not x_{i}} 2^{-n \varepsilon} \frac{p\left(x^{\prime}\right)}{p(x)}} \\
& =p_{\sigma_{n}}\left(\hat{B}^{(n)}\left(x_{i}, \varepsilon\right) \mid x_{i}\right) \frac{1}{\frac{p\left(c_{i}\right)}{p(x)}+2^{-n \varepsilon} C^{\prime}} \\
& \geq \frac{1-2^{-\frac{n}{k} \varepsilon} C\left(\frac{n}{k}+1\right)^{k|\mathcal{Y}|}}{\frac{p\left(c_{i}\right)}{p(x)}+2^{-n \varepsilon} C^{\prime}}
\end{aligned}
$$

where: (B.15) follows from the definition of $q_{i}^{n}(x)$ and an application of Bayes rule, and from the fact that $p_{\sigma_{n}}\left(y^{n} \mid x\right)=p_{\sigma_{n}}\left(y^{n} \mid x_{i}\right)$; ( $(\underline{\mathrm{B} .16})$ follows from a simple union bound and from Lemma B.1. (B.17) follows from the fact that, by assumption, $\hat{B}^{(n)}(x, 2 \varepsilon) \cap \hat{B}^{(n)}\left(x^{\prime}, 2 \varepsilon\right)=\emptyset$ (also note that $\left.\hat{B}^{(n)}(x, 2 \varepsilon)=\hat{B}^{(n)}\left(x_{i}, 2 \varepsilon\right)\right)$; (B.18) follows by definition of $\hat{B}^{(n)}(x, \varepsilon)=$ $\hat{B}^{(n)}\left(x_{i}, \varepsilon\right)$; here $C^{\prime}$ is a suitable constant, not depending on $n$; (B.19) follows from Lemma B.2.

Now, let $\left\{n_{j}\right\}$ be a sequence of indices such that, for each $x$ and $i, q_{i}^{n_{j}}(x)$ converges to a limit, say $L_{i}(x)$ (such a sub-sequence must exist, by Bolzano-Weierstrass.) The inequality

$$
q_{i}^{n}(x) \geq \frac{1-2^{-\frac{n}{k} \varepsilon} C\left(\frac{n}{k}+1\right)^{k|\mathcal{Y}|}}{\frac{p\left(c_{i}\right)}{p(x)}+2^{-n \varepsilon} C^{\prime}}
$$

which holds for each $n$ that is a multiple of $k$, implies that these limits satisfy $L_{i}(x) \geq$ $\frac{p(x)}{p\left(c_{i}\right)}$. Since point-wise convergence for each $x$ implies convergence of $q_{i}^{n_{j}}(\cdot)$ to a probability distribution, we have that, for each $i$ and $x$, actually equality must hold: $L_{i}(x)=\frac{p(x)}{p\left(c_{i}\right)}$. Thus, for each $i=1, \ldots, K, q_{i}^{n_{j}}(\cdot) \rightarrow p(\cdot \mid c)$, which proves (B.14).

\section{Appendix C. BACKWARd InduCtion}

We give some details of the derivation of an optimal strategy of length 2 for the system in Example 3.2. We take Shannon entropy as the chosen uncertainty measure. In Figure 9 we give a partial representation of the related MDP. According to the Backward Induction method, we compute the reward of each node, starting from the leaves of the MDP-tree and then propagating them up to the root. Let us denote by $R(n)$ the reward assigned to a node $n$. Applying the algorithm, we compute as follows (recall that levels are counted from the root, which has level 0 ).

- Level 4. The reward associated to each leaf node $n$ is $R(n)=0$ 


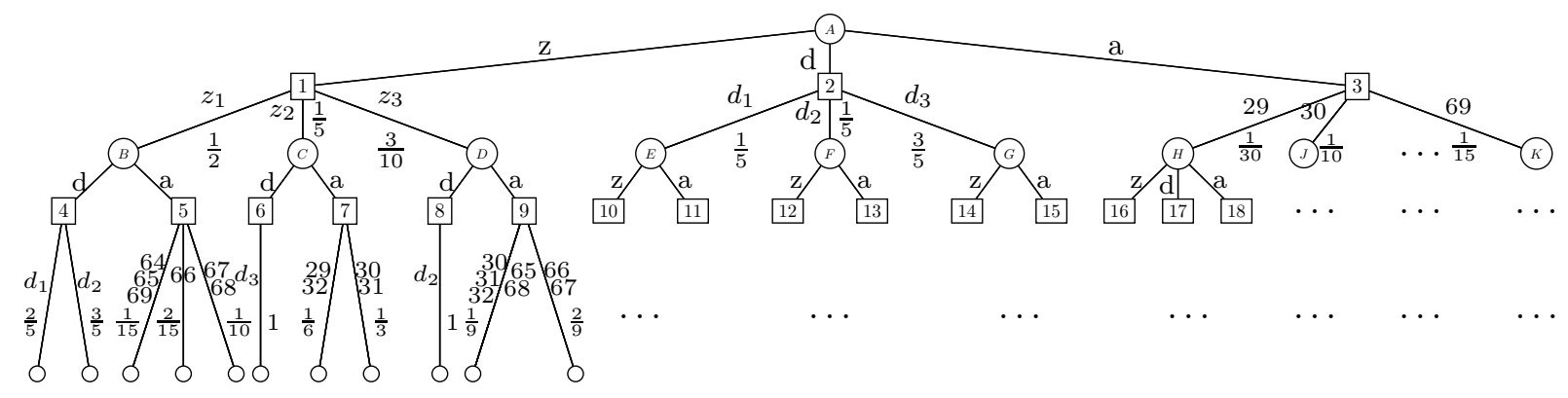

Figure 9. A partial representation of the MDP for Example 3.2 Here, the symbols 'z', 'd' and 'a' are abbreviations for actions $Z I P$, Date and Age, respectively. For simplicity, probability distributions labelling decision nodes are not shown. Moreover, leaves with the same labels and the same father, and the corresponding incoming arcs, have been coalesced.

- Level 3. Each probabilistic node, which is the $a$-child of a decision node $h$, receives as a reward the sum of its immediate gain, $I_{[a]}\left(\mathcal{S}, p^{h}\right)$, and the average reward of its children: $R(4)=R(5)=\log 5-\frac{2}{5}-\frac{3}{5} \log 3, R(6)=R(8)=0, R(7)=\frac{1}{3}, R(9)=\log 3-\frac{4}{3}$, and so on.

- Level 2. Each decision node receives as a reward the maximum of its children' rewards (and the corresponding action is recorded): $R(B)=\log 5-\frac{2}{5}-\frac{3}{5} \log 3, R(C)=\frac{1}{3}, R(D)=$ $\log 3-\frac{4}{3}$ and so on.

- Level 1. Each probabilistic node receives the following rewards: $R(1)=\log 10-\frac{9}{25}-$ $\frac{27}{50} \log 3 \approx 2.11, R(2)=\log 10-\frac{11}{15}-\frac{1}{5} \log 3 \approx 2.27$ and $R(3)=\log 10-\frac{3}{5}-\frac{1}{5} \log 3 \approx 2.4$.

- Level 0 . The decision node $A$ receives the reward $R(A)=R(3) \approx 2.4$

Taking into account the maximal children' rewards selected at each decision node, we have the following optimal strategy of length 2 (its tree representation is given in Figure 8.)

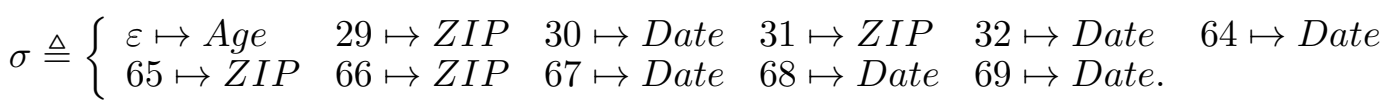

